TRANSACTIONS OF THE

AMERICAN MATHEMATICAL SOCIETY

Volume 364, Number 1, January 2012, Pages 195-232

S 0002-9947(2011)05358-4

Article electronically published on August 3, 2011

\title{
TRANSFER MAPS IN HOCHSCHILD (CO)HOMOLOGY AND APPLICATIONS TO STABLE AND DERIVED INVARIANTS AND TO THE AUSLANDER-REITEN CONJECTURE
}

\author{
STEFFEN KOENIG, YUMING LIU, AND GUODONG ZHOU
}

\begin{abstract}
Derived equivalences and stable equivalences of Morita type, and new (candidate) invariants thereof, between symmetric algebras will be investigated, using transfer maps as a tool. Close relationships will be established between the new invariants and the validity of the Auslander-Reiten conjecture, which states the invariance of the number of non-projective simple modules under stable equivalence. More precisely, the validity of this conjecture for a given pair of algebras, which are stably equivalent of Morita type, will be characterized in terms of data refining Hochschild homology (via Külshammer ideals) being invariant and also in terms of cyclic homology being invariant. Thus, validity of the Auslander-Reiten conjecture implies a whole set of ring theoretic and cohomological data to be invariant under stable equivalence of Morita type, and hence also under derived equivalence. We shall also prove that the Batalin-Vilkovisky algebra structure of Hochschild cohomology for symmetric algebras is preserved by derived equivalence. The main tools to be developed and used are transfer maps and their properties, in particular a crucial compatibility condition between transfer maps in Hochschild homology and Hochschild cohomology via the duality between them.
\end{abstract}

\section{INTRODUCTION}

Derived equivalences have been studied and used in representation theory of groups and of algebras since the pioneering work of Happel ([12, 13]), of Rickard ([28, 29, 30]) and of Keller ([15]). In particular, Broué's conjecture ([6]) has been the starting point of a major development in modular representation theory of finite groups, centreing around derived equivalences and their consequences. More generally, Broué ([7]) has introduced stable equivalences of Morita type, which are implied by derived equivalences between symmetric algebras. There are two key sets of problems about derived or stable equivalences. One is concerned with constructing derived or stable equivalences. The other one is about identifying and describing invariants, both of an abstract and of an explicit nature. It is this second complex of problems that we will address in this article.

Received by the editors January 29, 2010 and, in revised form, April 1, 2010.

2010 Mathematics Subject Classification. Primary 16G10, 16E40; Secondary 20C20.

Key words and phrases. Auslander-Reiten conjecture, derived equivalence, Hochschild (co)homology, stable equivalence of Morita type, transfer map.

The second author was supported by Marie Curie Fellowship IIF and by SRF for ROCS, SEM.

The third author benefited from financial support via a postdoctoral fellowship from the network "Representation theory of algebras and algebraic Lie theory" and the DAAD. This research work was mainly done while the last two authors visited the University of Köln.

(C)2011 American Mathematical Society Reverts to public domain 28 years from publication 
While derived equivalences have been shown to preserve various cohomological invariants such as Hochschild (co)homology ([30]), $K$-theory (33, 8]) and cyclic homology ([16]), much less is known for stable equivalences of Morita type. On the other hand, stable equivalences of Morita type are both more frequent and more explicit than derived equivalences. We propose to use transfer maps as a tool to set up and to investigate invariants under derived equivalences and under stable equivalences of Morita type. In the first part of this paper we will define the appropriate transfer maps and develop their basic properties, in particular a crucial compatibility condition (Theorem 2.10 and Corollary 2.12). In the second part we shall present some applications of the theory developed in the first part. We then will demonstrate the usefulness of the transfer maps in a general and abstract setup; we will work with symmetric algebras in full generality and with stable equivalences of Morita type in general, thus getting consequences for derived equivalences between group algebras, or blocks of group algebras, as well.

The consequences we will derive from properties of transfer maps are threefold. First, we will discuss countable series of potential invariants, which all can be seen as refining Hochschild homology, but which are of a ring theoretical nature. While Hochschild homology in positive degree is known to be invariant under stable equivalences of Morita type (23), Hochschild homology in degree zero is an invariant if and only if the two algebras in question have the same number of non-projective simple modules, that is, when the so-called Auslander-Reiten conjecture (2]) is valid in this situation (24]). The series of candidate invariants that we are considering have been defined by Külshammer $([18,19])$ in terms of ideals of the centre of an algebra, and thus are closely related to degree zero Hochschild homology. We will show that any of these data is an invariant under stable equivalence of Morita type if and only if the Auslander-Reiten conjecture is valid in this case (Corollary 4.6 and Proposition 5.8). The main tools to relate these data for different algebras are, of course, transfer maps.

Second, we will look at another potential invariant, cyclic homology. Here, the transfer maps come in via Connes' operator. Cyclic homology is a derived invariant by a result of Keller ([16]). Surprisingly, it turns out that in odd degrees it is also invariant under stable equivalence of Morita type, while in even degrees it is so if and only if the Auslander-Reiten conjecture is valid for the given equivalence (Theorem 9.6).

Thus, our results provide various new interpretations of and a new approach to the Auslander-Reiten conjecture (which at present appears to be far beyond reach), to be based on the equivalent versions of the conjecture contained in our results. To demonstrate feasibility of this approach, we give a sufficient criterion for the Auslander-Reiten conjecture to hold true. For two not necessarily symmetric algebras $A$ and $B$ over a field of positive characteristic, the conjecture follows from the existence of two stable equivalences of Morita type, one relating $A$ and $B$ and the other one relating their trivial extension algebras $\mathbb{T}(A)$ and $\mathbb{T}(B)$ (Corollary 8.2).

Third, our results imply extensions and new proofs of various known results, especially on derived categories. We reprove some results of Alexander Zimmermann ([35, 36, 37]) concerning Külshammer ideals using transfer maps. We also prove that the Batalin-Vilkovisky algebra structure ([34, 26]), in particular, the Gerstenhaber algebra structure, over Hochschild cohomology of symmetric algebras is preserved by a derived equivalence (Theorems 10.7 and 10.8). 
Now we are going to describe the contents of this article in more detail. In Section 2, we recall transfer maps in Hochschild homology defined by Bouc, and we introduce transfer maps in Hochschild cohomology for symmetric algebras. We prove the compatibility theorem between transfer maps in Hochschild homology and in Hochschild cohomology in this section. Section 3 studies when transfer maps preserve the product structure over Hochschild cohomology. $P$-power maps over zero-degree Hochschild homology groups are investigated in Section 4. Section 5 contains the stable version of Külshammer's $\zeta_{n}$. $P$-power maps over the centre $Z(A)$, Külshammer's maps $\kappa_{n}$ and their stable version are considered in the sixth section. Higher dimensional analogues are presented in the seventh section. Section 8 contains the trivial extension construction. Cyclic homology is studied in the ninth section and stable cyclic homology is introduced. We consider Batalin-Vilkovisky algebra structure in the last section.

\section{TRANSFER MAPS}

Let $k$ be a field of arbitrary characteristic and let $A$ be a finite dimensional $k$ algebra. In the sequel, $\otimes$ will denote $\otimes_{k}$. The bar $\operatorname{resolution} \operatorname{Bar}_{\bullet}(A)$ is defined as follows: $\operatorname{Bar}_{n}(A)=A^{\otimes(n+2)}$ and for $n \geq 1$, the differential is $d^{\prime}=\sum_{i=0}^{n}(-1)^{i} d_{i}^{\prime}$ : $\operatorname{Bar}_{n}(A)=A^{\otimes(n+2)} \rightarrow \operatorname{Bar}_{n-1}(A)=A^{\otimes(n+1)}$, where for $0 \leq i \leq n, d_{i}^{\prime}$ sends $a_{0} \otimes \cdots \otimes a_{n+1}$ for $a_{0}, \ldots, a_{n+1} \in A$ to

$$
a_{0} \otimes \cdots \otimes a_{i-1} \otimes a_{i} a_{i+1} \otimes a_{i+2} \otimes \cdots \otimes a_{n+1} .
$$

Then $\left(\operatorname{Bar}_{\bullet}(A), d^{\prime}\right)$ is a projective resolution of $A$ as $A^{e}=A \otimes_{k} A^{o p}$-modules. Let $(C \bullet(A), d)=\left(A \otimes_{A^{e}} \operatorname{Bar}(A), I d_{A} \otimes d^{\prime}\right)$ be the Hochschild complex. Namely, for $n \geq 0, C_{n}(A) \cong A^{\otimes(n+1)}$ and for $n \geq 1$ the differential $d: C_{n}(A) \rightarrow C_{n-1}(A)$ sends $a_{0} \otimes \cdots \otimes a_{n}$ with $a_{0}, \ldots, a_{n} \in A$ to

$$
\sum_{i=0}^{n-1}(-1)^{i} a_{0} \otimes \cdots \otimes a_{i} a_{i+1} \otimes \cdots \otimes a_{n}+(-1)^{n} a_{n} a_{0} \otimes a_{1} \otimes \cdots \otimes a_{n-1} .
$$

Next we recall the construction of transfer maps in Hochschild homology due to Bouc (4). Transfer maps in Hochschild homology and in cyclic homology also have been studied by Keller ([16]) and by Loday ([25]). Recall that for an algebra $A$, the Hochschild homology of degree zero is $H H_{0}(A)=A / K(A)$, where $K(A)$ is the subspace spanned by commutators.

Let $A$ and $B$ be two finite dimensional $k$-algebras and let $M$ be an $A$ - $B$-bimodule such that $M$ is finitely generated and projective as a right $B$-module. There exist $x_{i} \in M$ and $\varphi_{i} \in \operatorname{Hom}_{B}(M, B)$ with $1 \leq i \leq s$ such that for any $x \in M, x=$ $\sum_{i} x_{i} \varphi_{i}(x)$. Then one can define a transfer map $t_{M}: H H_{n}(A) \rightarrow H H_{n}(B)$ for any $n \geq 0$. The construction on the level of Hochschild complexes is as follows: $t_{M}: C_{n}(A) \rightarrow C_{n}(B)$ sends $a_{0} \otimes a_{1} \otimes \cdots \otimes a_{n}$ to

$$
\sum_{1 \leq i_{0}, \ldots, i_{n} \leq s} \varphi_{i_{0}}\left(a_{0} x_{i_{1}}\right) \otimes \varphi_{i_{1}}\left(a_{1} x_{i_{2}}\right) \otimes \cdots \otimes \varphi_{i_{n}}\left(a_{n} x_{i_{0}}\right) .
$$

Bouc proved that this is a chain map and thus induces a morphism between Hochschild homology groups, written also by $t_{M}: H H_{n}(A) \rightarrow H H_{n}(B)$. In particular, the construction in degree zero

$$
t_{M}: H H_{0}(A)=A / K(A) \rightarrow H H_{0}(B)=B / K(B)
$$

is given by $a+K(A) \mapsto \sum_{i=1}^{s} \varphi_{i}\left(a x_{i}\right)+K(B)$. 
We summarize basic properties of this transfer map in the following

Proposition 2.1 ([4, Section 3]). Let $A, B$ and $C$ be finite dimensional $k$-algebras.

(1) If $M$ is an $A$-B-bimodule and $N$ is a $B$-C-bimodule such that $M_{B}$ and $N_{C}$ are finitely generated and projective, then $t_{N} \circ t_{M}=t_{M \otimes_{B} N}: H H_{n}(A) \rightarrow H H_{n}(C)$, for each $n \geq 0$.

(2) Let

$$
0 \rightarrow L \rightarrow M \rightarrow N \rightarrow 0
$$

be a short exact sequence of $A$-B-bimodules which are finitely generated and projective as right $B$-modules. Then $t_{M}=t_{L}+t_{N}: H H_{n}(A) \rightarrow H H_{n}(B)$, for each $n \geq 0$.

(3) For a finitely generated projective $A$-B-bimodule $P$, the transfer map $t_{P}$ : $H H_{n}(A) \rightarrow H H_{n}(B)$ is zero for each $n>0$.

(4) Consider $A$ as an $A$-A-bimodule by left and right multiplication, then $t_{A}$ : $\mathrm{HH}_{n}(A) \rightarrow \mathrm{HH}_{n}(A)$ is the identity map for any $n \geq 0$.

Remark 2.2. Given a bounded (cochain) complex $X^{\bullet}$ of $A$-B-bimodules whose terms are finitely generated and projective as right $B$-modules, one can also define a transfer map $t_{X} \bullet: H H_{n}(A) \rightarrow H H_{n}(B)$ by $t_{X} \bullet:=\sum_{i}(-1)^{i} t_{X^{i}}$. Note that if $Y^{\bullet}$ is another bounded complex of $A$-B-bimodules whose terms are finitely generated and projective as right $B$-modules such that $X^{\bullet}$ and $Y^{\bullet}$ are quasi-isomorphic, then $t_{X} \bullet=t_{Y} \bullet([4$, Section 4]).

We will define transfer maps in Hochschild cohomology for symmetric algebras. Our definition will turn out to coincide with the construction due to Linckelmann ([20]). We recall some basic facts about symmetric algebras, and for details we refer to [20, Section 6]. A symmetric algebra is a finite dimensional $k$-algebra such that there is a symmetric non-degenerate associative bilinear form $(,)_{A}: A \times A \rightarrow k$, or equivalently, $A \cong D(A)=A^{*}=\operatorname{Hom}_{k}(A, k)$ as bimodules. The image of the unit element of $A$ under this isomorphism is called a symmetrizing form on $A$ and is denoted by $s$. Note that the bilinear form can be given by $\left(a, a^{\prime}\right)_{A}=s\left(a a^{\prime}\right)$ for arbitrary $a, a^{\prime} \in A$.

Now let $B$ be another finite dimensional $k$-algebra and let ${ }_{A} M_{B}$ be an $A$ $B$-bimodule. Then $M^{*}=\operatorname{Hom}_{k}(M, k)$ is isomorphic to $\operatorname{Hom}_{A}(M, A)$ as $B$ - $A$ bimodules. The isomorphism $\operatorname{Hom}_{A}(M, A) \cong M^{*}$ sends $f \in \operatorname{Hom}_{A}(M, A)$ to the composition $s \circ f$. The inverse map can be described as follows. Let $\left\{u_{i}\right\}$ be a basis of $A$ and let $\left\{v_{i}\right\}$ be the dual basis with respect to the bilinear form $(,)_{A}$ or $s \in A^{*}$, that is, $\left(u_{i}, v_{j}\right)=\delta_{i j}$. Then the image of $\theta \in M^{*}$ under the inverse map is the map sending $x \in M$ to $\sum_{i} \theta\left(v_{i} x\right) u_{i}$.

Now suppose that ${ }_{A} M$ is finitely generated and projective. Then we have isomorphisms of functors

$$
\operatorname{Hom}_{A}(M,-) \cong \operatorname{Hom}_{A}(M, A) \otimes_{A}-\cong M^{*} \otimes_{A}-.
$$

By adjointness, there is an adjoint pair $\left(M \otimes_{B}-, M^{*} \otimes_{A}-\right)$. One can compute the associate counit morphism $\varepsilon_{M}: M \otimes_{B} M^{*} \rightarrow A$. Let $x \in M$ and $\theta \in M^{*}$. Then $\varepsilon_{M}\left(x \otimes_{A} \theta\right)=\sum_{i} \theta\left(v_{i} x\right) u_{i}$.

Now suppose that $B$ is also symmetric with $t \in B^{*}$ giving the symmetrizing form. If, furthermore, $M_{B}$ is finitely generated and projective, then $M^{*}$ is finitely generated projective as a left $B$-module and we have an isomorphism of functors

$$
\operatorname{Hom}_{B}\left(M^{*},-\right) \cong \operatorname{Hom}_{B}\left(M^{*}, B\right) \otimes_{B}-\cong M^{* *} \otimes_{B}-\cong M \otimes_{B}-
$$


Hence there is another adjoint pair $\left(M^{*} \otimes_{A}-, M \otimes_{B}-\right)$. Its unit morphism $\eta_{M^{*}}$ : $A \rightarrow M \otimes_{B} M^{*}$ can be computed. Since $M$ is finitely generated and projective as a right $B$-module, there exist $x_{i} \in M$ and $\varphi_{i} \in \operatorname{Hom}_{B}(M, B)$ with $1 \leq i \leq s$ such that for any $x \in M, x=\sum_{i} x_{i} \varphi_{i}(x)$. It follows that $\eta_{M^{*}}$ sends $a \in A$ to $\sum_{i} a x_{i} \otimes_{B} t \circ \varphi_{i}$.

Remark 2.3. We can give another realization of the above unit morphism $\eta_{M^{*}}$ by considering adjoint pairs between the categories of right modules. Namely, since $M$ is finitely generated and projective as a right $B$-module, there are isomorphisms of functors

$$
\operatorname{Hom}_{B}(M,-) \cong-\otimes_{B} \operatorname{Hom}_{B}(M, B) \cong-\otimes_{B} M^{*} .
$$

Hence there is an adjoint pair $\left(-\otimes_{A} M,-\otimes_{B} M^{*}\right)$. A computation shows that the unit morphism $A \rightarrow M \otimes_{B} M^{*}$ of this adjoint pair coincides with the above $\eta_{M^{*}}$.

Now transfer maps in Hochschild cohomology can be defined. Recall that the Hochschild cohomology is the cohomology of the Hochschild complex $C^{\bullet}(A)=$ $\operatorname{Hom}_{A^{e}}\left(\operatorname{Bar}_{\bullet}(A), A\right)$. Then $C^{n}(A)=\operatorname{Hom}_{A^{e}}\left(\operatorname{Bar}_{n}(A), A\right)=\operatorname{Hom}_{k}\left(A^{\otimes n}, A\right)$. Our goal is to define a chain map $t^{M}: C^{\bullet}(B) \rightarrow C^{\bullet}(A)$ for an $A$-B-bimodule ${ }_{A} M_{B}$ which is finitely generated and projective as a left $A$-module and a right $B$-module for two symmetric algebras $A$ and $B$. To this end, we need a chain map

$$
\Theta_{\bullet}: \operatorname{Bar}_{\bullet}(A) \rightarrow M \otimes_{B} \operatorname{Bar}_{\bullet}(B) \otimes_{B} M^{*},
$$

which lifts the unit morphism $\eta_{M^{*}}: A \rightarrow M \otimes_{B} M^{*}$.

Proposition 2.4. Let $A$ and $B$ be two finite dimensional $k$-algebras with $B$ symmetric by a symmetrizing form $t \in B^{*}$. Let ${ }_{A} M_{B}$ be an $A$-B-bimodule such that $M_{B}$ is finitely generated and projective.

Then for $n \geq 0$, the map

$$
\begin{aligned}
& \Theta_{n}: \operatorname{Bar}_{n}(A)=A^{\otimes(n+2)} \rightarrow M \otimes_{B} \operatorname{Bar}_{n}(B) \otimes_{B} M^{*}=M \otimes B^{\otimes n} \otimes M^{*} \\
& \text { sending } a_{0} \otimes \cdots \otimes a_{n+1} \text { for } a_{0}, \ldots, a_{n+1} \in A \text { to } \\
& \sum_{i_{0}, \ldots, i_{n}} a_{0} x_{i_{1}} \otimes \varphi_{i_{1}}\left(a_{1} x_{i_{2}}\right) \otimes \cdots \otimes \varphi_{i_{n}}\left(a_{n} x_{i_{0}}\right) \otimes t \circ \varphi_{i_{0}} a_{n+1}
\end{aligned}
$$

commutes with the differential, where $x_{i} \in M$ and $\varphi_{i} \in \operatorname{Hom}_{B}(M, B)$ with $1 \leq i \leq s$ such that for any $x \in M, x=\sum_{i} x_{i} \varphi_{i}(x)$. Moreover, $\Theta_{\bullet}$ lifts the unit morphism $\eta_{M^{*}}: A \rightarrow M \otimes_{B} M^{*}$.

Proof. We first prove that $\Theta_{\bullet}$ lifts the unit morphism $\eta_{M^{*}}: A \rightarrow M \otimes_{B} M^{*}$, that is, $\eta_{M^{*}} \mu_{A}=\left(I d_{M} \otimes \mu_{B} \otimes I d_{M^{*}}\right) \Theta_{0}$, where $\mu_{A}: A \otimes_{k} A \rightarrow A$ and $\mu_{B}: B \otimes_{k} B \rightarrow B$ are the multiplication maps. For $a_{0}, a_{1} \in A$, we have $\left(I d_{M} \otimes \mu_{B} \otimes I d_{M^{*}}\right) \Theta_{0}\left(a_{0} \otimes a_{1}\right)=$ $\left(I d_{M} \otimes \mu_{B} \otimes I d_{M^{*}}\right)\left(\sum_{i} a_{0} x_{i} \otimes t \circ \varphi_{i} a_{1}\right)=\sum_{i} a_{0} x_{i} \otimes_{B} t \circ \varphi_{i} a_{1}$, and on the other hand, $\eta_{M^{*}} \mu_{A}\left(a_{0} \otimes a_{1}\right)=\eta_{M^{*}}\left(a_{0} a_{1}\right)=\sum_{i} a_{0} x_{i} \otimes_{B} t \circ \varphi_{i} a_{1}$ since $\eta_{M^{*}}$ is an $A$ - $A$-bimodule homomorphism.

Next, we prove that $\Theta_{\bullet}$ is a chain map. To do this, we need to prove that $\Theta_{n-1} d_{i}^{\prime}=\left(I d_{M} \otimes d_{i}^{\prime} \otimes I d_{M^{*}}\right) \Theta_{n}$ for each $0 \leq i \leq n$. We shall only give the proof for $i=0$ and for $i=n$, the other cases being similar. 
Case $i=0$. For $a_{0}, \ldots, a_{n+1} \in A$, we have

$$
\begin{aligned}
& \Theta_{n-1} d_{0}^{\prime}\left(a_{0} \otimes \cdots \otimes a_{n+1}\right) \\
= & \Theta_{n-1}\left(a_{0} a_{1} \otimes \cdots \otimes a_{n+1}\right) \\
= & \sum_{i_{2}, \ldots, i_{n}, i_{0}} a_{0} a_{1} x_{i_{2}} \otimes \varphi_{i_{2}}\left(a_{2} x_{i_{3}}\right) \otimes \cdots \otimes \varphi_{i_{n}}\left(a_{n} x_{i_{0}}\right) \otimes t \circ \varphi_{i_{0}} a_{n+1},
\end{aligned}
$$

and on the other hand we have

$$
\begin{aligned}
& \left(I d_{M} \otimes d_{0}^{\prime} \otimes I d_{M^{*}}\right) \Theta_{n}\left(a_{0} \otimes \cdots \otimes a_{n+1}\right) \\
= & \left(I d_{M} \otimes d_{0}^{\prime} \otimes I d_{M^{*}}\right) \\
& \left(\sum_{i_{0}, i_{1}, i_{2}, \ldots, i_{n}} a_{0} x_{i_{1}} \otimes \varphi_{i_{1}}\left(a_{1} x_{i_{2}}\right) \otimes \cdots \otimes \varphi_{i_{n}}\left(a_{n} x_{i_{0}}\right) \otimes t \circ \varphi_{i_{0}} a_{n+1}\right) \\
= & \sum_{i_{0}, i_{1}, i_{2}, \ldots, i_{n}} a_{0} x_{i_{1}} \varphi_{i_{1}}\left(a_{1} x_{i_{2}}\right) \otimes \varphi_{i_{2}}\left(a_{2} x_{i_{3}}\right) \cdots \otimes \varphi_{i_{n}}\left(a_{n} x_{i_{0}}\right) \otimes t \circ \varphi_{i_{0}} a_{n+1} \\
= & \sum_{i_{0}, i_{2}, \ldots, i_{n}}\left(\sum_{i_{1}} a_{0} x_{i_{1}} \varphi_{i_{1}}\left(a_{1} x_{i_{2}}\right)\right) \otimes \varphi_{i_{2}}\left(a_{2} x_{i_{3}}\right) \cdots \otimes \varphi_{i_{n}}\left(a_{n} x_{i_{0}}\right) \otimes t \circ \varphi_{i_{0}} a_{n+1} \\
= & \sum_{i_{2}, \ldots, i_{n}, i_{0}} a_{0} a_{1} x_{i_{2}} \otimes \varphi_{i_{2}}\left(a_{2} x_{i_{3}}\right) \otimes \cdots \otimes \varphi_{i_{n}}\left(a_{n} x_{i_{0}}\right) \otimes t \circ \varphi_{i_{0}} a_{n+1},
\end{aligned}
$$

where we use the equality

$$
\sum_{i_{1}} x_{i_{1}} \varphi_{i_{1}}\left(a_{1} x_{i_{2}}\right)=a_{1} x_{i_{2}}
$$

Case $i=n$. For $a_{0}, \ldots, a_{n+1} \in A$, we have

$$
\begin{aligned}
& \Theta_{n-1} d_{n}^{\prime}\left(a_{0} \otimes \cdots \otimes a_{n+1}\right) \\
= & \Theta_{n-1}\left(a_{0} \otimes \cdots \otimes a_{n} a_{n+1}\right) \\
= & \sum_{i_{0}, i_{1}, \ldots, i_{n-1}} a_{0} x_{i_{1}} \otimes \varphi_{i_{1}}\left(a_{1} x_{i_{2}}\right) \otimes \cdots \otimes \varphi_{i_{n-1}}\left(a_{n-1} x_{i_{0}}\right) \otimes t \circ \varphi_{i_{0}} a_{n} a_{n+1},
\end{aligned}
$$

and on the other hand we have

$$
\begin{aligned}
& \left(I d_{M} \otimes d_{n}^{\prime} \otimes I d_{M^{*}}\right) \Theta_{n}\left(a_{0} \otimes \cdots \otimes a_{n+1}\right) \\
= & \left(I d_{M} \otimes d_{n}^{\prime} \otimes I d_{M^{*}}\right) \\
& \left(\sum_{i_{0}, \ldots, i_{n}} a_{0} x_{i_{1}} \otimes \varphi_{i_{1}}\left(a_{1} x_{i_{2}}\right) \otimes \cdots \otimes \varphi_{i_{n}}\left(a_{n} x_{i_{0}}\right) \otimes t \circ \varphi_{i_{0}} a_{n+1}\right) \\
= & \sum_{i_{0}, \ldots, i_{n}} a_{0} x_{i_{1}} \otimes \varphi_{i_{1}}\left(a_{1} x_{i_{2}}\right) \otimes \cdots \otimes \varphi_{i_{n-1}}\left(a_{n-1} x_{i_{n}}\right) \otimes \varphi_{i_{n}}\left(a_{n} x_{i_{0}}\right) t \circ \varphi_{i_{0}} a_{n+1} \\
= & \sum_{i_{n}, i_{1}, \ldots, i_{n-1}, i_{0}} a_{0} x_{i_{1}} \otimes \varphi_{i_{1}}\left(a_{1} x_{i_{2}}\right) \\
& \otimes \cdots \otimes \varphi_{i_{n-1}}\left(a_{n-1} x_{i_{0}}\right) \otimes \varphi_{i_{0}}\left(a_{n} x_{i_{n}}\right) t \circ \varphi_{i_{n}} a_{n+1} \\
= & \sum_{i_{0}, i_{1}, \ldots, i_{n-1}} a_{0} x_{i_{1}} \otimes \cdots \otimes \varphi_{i_{n-1}}\left(a_{n-1} x_{i_{0}}\right) \otimes\left(\sum_{i_{n}} \varphi_{i_{0}}\left(a_{n} x_{i_{n}}\right) t \circ \varphi_{i_{n}} a_{n+1}\right) .
\end{aligned}
$$

To finish the proof, we need to show that

$$
t \circ \varphi_{i_{0}} a_{n} a_{n+1}=\sum_{i_{n}} \varphi_{i_{0}}\left(a_{n} x_{i_{n}}\right) t \circ \varphi_{i_{n}} a_{n+1} .
$$


For $x \in M$, we have $t \circ \varphi_{i_{0}} a_{n} a_{n+1}(x)=t\left(\varphi_{i_{0}}\left(a_{n} a_{n+1} x\right)\right)$, and on the other hand we have

$$
\begin{aligned}
\sum_{i_{n}} \varphi_{i_{0}}\left(a_{n} x_{i_{n}}\right) t \circ \varphi_{i_{n}} a_{n+1}(x)=\sum_{i_{n}} t \circ \varphi_{i_{n}}\left(a_{n+1} x \varphi_{i_{0}}\left(a_{n} x_{i_{n}}\right)\right) \\
=t\left(\sum_{i_{n}} \varphi_{i_{n}}\left(a_{n+1} x\right) \varphi_{i_{0}}\left(a_{n} x_{i_{n}}\right)\right)=t\left(\sum_{i_{n}} \varphi_{i_{0}}\left(a_{n} x_{i_{n}}\right) \varphi_{i_{n}}\left(a_{n+1} x\right)\right) \\
=t\left(\varphi_{i_{0}}\left(a_{n} \sum_{i_{n}} x_{i_{n}} \varphi_{i_{n}}\left(a_{n+1} x\right)\right)\right)=t\left(\varphi_{i_{0}}\left(a_{n} a_{n+1} x\right)\right) .
\end{aligned}
$$

Now we can define transfer maps in Hochschild cohomology for symmetric algebras. Let $A$ and $B$ be two symmetric algebras and let ${ }_{A} M_{B}$ be a bimodule such that ${ }_{A} M$ and $M_{B}$ are finitely generated and projective. Then for $f \in C^{n}(B)=$ $\operatorname{Hom}_{B^{e}}\left(\operatorname{Bar}_{n}(B), B\right)$ with $n \geq 0$, we define $\operatorname{tr}^{M}(f)$ to be the composition

$$
\operatorname{Bar}_{n}(A) \stackrel{\Theta_{n}}{\rightarrow} M \otimes_{B} \operatorname{Bar}_{n}(B) \otimes_{B} M^{*} \stackrel{I d_{M} \otimes f \otimes I d_{M^{*}}}{\longrightarrow} M \otimes_{B} B \otimes_{B} M^{*} \stackrel{\varepsilon_{M}}{\longrightarrow} A .
$$

Proposition 2.5. (1) For $f \in C^{n}(B)=\operatorname{Hom}_{B^{e}}\left(\operatorname{Bar}_{n}(B), B\right) \cong \operatorname{Hom}_{k}\left(B^{\otimes n}, B\right)$ with $n \geq 0$, the map $\operatorname{tr}^{M}(f) \in \operatorname{Hom}_{k}\left(A^{\otimes n}, A\right)$ sends $a_{1} \otimes \cdots \otimes a_{n}$ to

$$
\sum_{i_{0}, \ldots, i_{n}, j}\left(\varphi_{i_{0}}\left(v_{j} x_{i_{1}}\right), f\left(\varphi_{i_{1}}\left(a_{1} x_{i_{2}}\right) \otimes \cdots \otimes \varphi_{i_{n}}\left(a_{n} x_{i_{0}}\right)\right)\right)_{B} u_{j},
$$

where $x_{i} \in M$ and $\varphi_{i} \in \operatorname{Hom}_{B}(M, B)$ with $1 \leq i \leq s$ such that for any $x \in M, x=\sum_{i} x_{i} \varphi_{i}(x)$, where $(,)_{B}$ is the bilinear form over $B$ and where $\left\{u_{j}\right\},\left\{v_{j}\right\}$ are dual bases in $A$, that is, $\left(u_{i}, v_{j}\right)_{A}=\delta_{i j}$.

(2) The map $\operatorname{tr}^{M}: C^{n}(B) \rightarrow C^{n}(A)$ is a chain map and thus induces a transfer map $t^{M}: H H^{n}(B) \rightarrow H H^{n}(A)$ for $n \geq 0$. In particular, in degree zero, $t^{M}: Z(B) \rightarrow Z(A)$ is given by

$$
b \mapsto \sum_{i, j}\left(\varphi_{i}\left(v_{j} x_{i}\right), b\right)_{B} u_{j} .
$$

Moreover, if we identify $Z(A)$ with $\operatorname{End}_{A^{e}}(A, A)\left(Z(B)\right.$ with $\operatorname{End}_{B^{e}}(B, B)$, respectively), then $t^{M}: Z(B) \rightarrow Z(A)$ coincides with the composition

$$
A \stackrel{\eta_{M^{*}}}{\rightarrow} M \otimes_{B} B \otimes_{B} M^{*} \stackrel{I d_{M} \otimes f \otimes I d_{M^{*}}}{\longrightarrow} M \otimes_{B} B \otimes_{B} M^{*} \stackrel{\varepsilon_{M}}{\rightarrow} A .
$$

Proof. The proof of the first assertion is easy using the explicit construction of $\Theta_{n}$ and $\varepsilon_{M}$. The second assertion is also direct since $\Theta_{\bullet}$ is a chain map by Proposition 2.4 .

Remark 2.6. (1) In 20] Linckelmann introduced transfer maps for symmetric algebras as follows. Let $A$ and $B$ be two symmetric $k$-algebras. Let ${ }_{A} M_{B}$ be an $A$ - $B$-bimodule such that ${ }_{A} M$ and $M_{B}$ are finitely generated and projective. Let $\mathcal{P}_{A}$ (resp. $\mathcal{P}_{B}$ ) be a projective resolution of $A$ (resp. of $B$ ) as bimodules. Suppose we are given $\zeta \in H H^{n}(B) \cong \operatorname{Hom}_{K\left(B^{e}\right)}\left(\mathcal{P}_{B}, \mathcal{P}_{B}[n]\right)$, where $K\left(B^{e}\right)$ is the homotopy category of complexes of $B^{e}$-modules. Then we define $t^{M}(\zeta) \in H H^{n}(A) \cong$ $\operatorname{Hom}_{K\left(A^{e}\right)}\left(\mathcal{P}_{A}, \mathcal{P}_{A}[n]\right)$ to be the class in the homotopy category $K\left(A^{e}\right)$ of the composition

$$
\mathcal{P}_{A} \rightarrow M \otimes_{B} \mathcal{P}_{B} \otimes_{B} M^{*} \stackrel{I d_{M} \otimes \zeta \otimes I d_{M^{*}}}{\longrightarrow} M \otimes_{B} \mathcal{P}_{B}[n] \otimes_{B} M^{*} \rightarrow \mathcal{P}_{A}[n] .
$$

Here the first map in the composition lifts the unit morphism $\eta_{M^{*}}: A \rightarrow M \otimes_{B} M^{*}$ and the third map lifts a translation of the counit morphism $\varepsilon_{M}: M \otimes_{B} M^{*} \rightarrow A$. 
It is obvious that our construction is a special case of Linckelmann's construction. We choose $\mathcal{P}_{A}\left(\right.$ resp. $\left.\mathcal{P}_{B}\right)$ to be the $\operatorname{Bar}$ resolution $\operatorname{Bar} \bullet(A)\left(\operatorname{resp} . \operatorname{Bar}_{\bullet}(B)\right)$ and we explicitly construct the first lift. Linckelmann's construction also works for a bounded complex $X^{\bullet}$ of $A$-B-bimodules whose terms are finitely generated and projective as left and right modules. But here we first define the case of modules and point out some basic properties of transfer maps. Afterwards we will deal with the case of complexes in Remark 2.8

(2) As Linckelmann pointed out in [20, Remark 2.10], the definition of the transfer map in Hochschild cohomology depends on the choice of the symmetrizing forms $s$ on $A$ and $t$ on $B$ in the following way. If $s^{\prime} \in A^{*}$ and $t^{\prime} \in B^{*}$ are some other symmetrizing forms, there are unique invertible elements $u \in Z(A)$ and $v \in Z(B)$ such that $s^{\prime}=u s$ and $t^{\prime}=v t$. It follows that the corresponding transfer map $t^{M^{\prime}}$ associated with $s^{\prime}$ and $t^{\prime}$ satisfies $t^{M^{\prime}}([f])=u^{-1} t^{M}(v[f])$ for any $[f] \in H H^{n}(B)$.

As in Hochschild homology, the transfer maps in Hochschild cohomology satisfy the following properties proved in [20]. Here we shall give a different proof for them by using our explicit construction and some ideas from [4].

Proposition 2.7 ([20, Section 2]). Let $A, B$ and $C$ be (finite dimensional) symmetric $k$-algebras.

(1) If $M$ is an $A$-B-bimodule and $N$ is a $B$-C-bimodule such that ${ }_{A} M, M_{B}$, ${ }_{B} N$ and $N_{C}$ are finitely generated and projective as left and right modules, then $t^{M} \circ t^{N}=t^{M \otimes_{B} N}: H H^{n}(C) \rightarrow H H^{n}(A)$, for each $n \geq 0$.

(2) Let

$$
0 \rightarrow L \stackrel{\alpha}{\rightarrow} M \stackrel{\beta}{\rightarrow} N \rightarrow 0
$$

be a short exact sequence of $A$-B-bimodules which are finitely generated and projective as left $A$-modules and as right $B$-modules. Then $t^{M}=t^{L}+t^{N}: H H^{n}(B) \rightarrow$ $H H^{n}(A)$, for each $n \geq 0$.

(3) For a finitely generated projective $A$-B-bimodule $P$, the transfer map $t^{P}$ : $H H^{n}(B) \rightarrow H H^{n}(A)$ is zero for each $n>0$.

(4) Consider $A$ as an $A$-A-bimodule by left and right multiplication. Then $t^{A}$ : $H H^{n}(A) \rightarrow H H^{n}(A)$ is the identity map for any $n \geq 0$.

Proof. (1) Since $M$ is finitely generated and projective as a right $B$-module, there exist $x_{i} \in M$ and $\varphi_{i} \in \operatorname{Hom}_{B}(M, B)$ with $1 \leq i \leq s$ such that for any $x \in M$, $x=\sum_{i} x_{i} \varphi_{i}(x)$. Similarly, since $N$ is finitely generated and projective as a right $C$-module, there exist $y_{j} \in N$ and $\psi_{j} \in \operatorname{Hom}_{C}(N, C)$ with $1 \leq j \leq t$ such that for any $y \in N, y=\sum_{j} y_{j} \psi_{j}(y)$. Moreover, we choose the elements $x_{i} \otimes y_{j} \in M \otimes_{B} N$ and define $\theta_{i, j} \in \operatorname{Hom}_{C}\left(M \otimes_{B} N, C\right)$ by $\theta_{i, j}(x \otimes y)=\psi_{j}\left(\varphi_{i}(x) y\right)$, where $1 \leq i \leq s$ and $1 \leq j \leq t$. Then for any $x \otimes y \in M \otimes_{B} N, \sum_{i, j}\left(x_{i} \otimes y_{j}\right) \theta_{i, j}(x \otimes y)=$ $\sum_{i, j} x_{i} \otimes y_{j} \psi_{j}\left(\varphi_{i}(x) y\right)=\sum_{i} x_{i} \otimes \varphi_{i}(x) y=\sum_{i} x_{i} \varphi_{i}(x) \otimes y=x \otimes y$. Since $A, B$ and $C$ are symmetric $k$-algebras, we can choose bilinear forms $(,)_{A},(,)_{B}$, and $(,)_{C}$, respectively. We choose dual bases $\left\{u_{i}\right\},\left\{v_{i}\right\}$ in $A$, that is, $\left(u_{i}, v_{j}\right)_{A}=\delta_{i j}$. Similarly, we choose dual bases $\left\{p_{j}\right\},\left\{q_{j}\right\}$ in $B$. To prove the statement, it suffices to prove that, for any $f \in \operatorname{Hom}_{k}\left(C^{\otimes n}, C\right), \operatorname{tr}^{M} \circ \operatorname{tr}^{N}(f)=\operatorname{tr}^{M \otimes_{B} N}(f)$.

For $f \in \operatorname{Hom}_{k}\left(C^{\otimes n}, C\right)$, we have $\operatorname{tr}^{N}(f) \in \operatorname{Hom}_{k}\left(B^{\otimes n}, B\right)$ sends $b_{1} \otimes \cdots \otimes b_{n}$ to

$$
\sum_{j_{0}, \ldots, j_{n}, j}\left(\psi_{j_{0}}\left(q_{j} y_{j_{1}}\right), f\left(\psi_{j_{1}}\left(b_{1} y_{j_{2}}\right) \otimes \cdots \otimes \psi_{j_{n}}\left(b_{n} y_{j_{0}}\right)\right)\right)_{C} p_{j}
$$


Let $g=\operatorname{tr}^{N}(f)$. Then we have that $\operatorname{tr}^{M}(g)=\operatorname{tr}^{M} \operatorname{tr}^{N}(f) \in \operatorname{Hom}_{k}\left(A^{\otimes n}, A\right)$ sends $a_{1} \otimes \cdots \otimes a_{n}$ to

$$
\begin{aligned}
\sum_{i_{0}, \ldots, i_{n}, i} & \left(\varphi_{i_{0}}\left(v_{i} x_{i_{1}}\right), g\left(\varphi_{i_{1}}\left(a_{1} x_{i_{2}}\right) \otimes \cdots \otimes \varphi_{i_{n}}\left(a_{n} x_{i_{0}}\right)\right)\right)_{B} u_{i} \\
= & \sum_{i_{0}, \ldots, i_{n}, i}\left(\varphi_{i_{0}}\left(v_{i} x_{i_{1}}\right), \sum_{j_{0}, \ldots, j_{n}, j}\left(\psi_{j_{0}}\left(q_{j} y_{j_{1}}\right), f\left(\psi_{j_{1}}\left(\varphi_{i_{1}}\left(a_{1} x_{i_{2}}\right) y_{j_{2}}\right)\right.\right.\right. \\
& \left.\left.\left.\otimes \cdots \otimes \psi_{j_{n}}\left(\varphi_{i_{n}}\left(a_{n} x_{i_{0}}\right) y_{j_{0}}\right)\right)\right)_{C} p_{j}\right)_{B} u_{i} \\
= & \sum_{i_{0}, \ldots, i_{n}, j_{0}, \ldots, j_{n}, i} \sum_{j}\left(\varphi_{i_{0}}\left(v_{i} x_{i_{1}}\right),\left(\psi_{j_{0}}\left(q_{j} y_{j_{1}}\right), f\left(\psi_{j_{1}}\left(\varphi_{i_{1}}\left(a_{1} x_{i_{2}}\right) y_{j_{2}}\right)\right.\right.\right. \\
& \left.\left.\left.\otimes \cdots \otimes \psi_{j_{n}}\left(\varphi_{i_{n}}\left(a_{n} x_{i_{0}}\right) y_{j_{0}}\right)\right)\right)_{C} p_{j}\right)_{B} u_{i} \\
= & \sum_{i_{0}, \ldots, i_{n}, j_{0}, \ldots, j_{n}, i} \sum_{j}\left(\psi_{j_{0}}\left(q_{j} y_{j_{1}}\right), f\left(\psi_{j_{1}}\left(\varphi_{i_{1}}\left(a_{1} x_{i_{2}}\right) y_{j_{2}}\right)\right.\right. \\
& \left.\left.\otimes \cdots \otimes \psi_{j_{n}}\left(\varphi_{i_{n}}\left(a_{n} x_{i_{0}}\right) y_{j_{0}}\right)\right)\right)_{C}\left(\varphi_{i_{0}}\left(v_{i} x_{i_{1}}\right), p_{j}\right)_{B} u_{i} \\
= & \sum_{i_{0}, \ldots, i_{n}, j_{0}, \ldots, j_{n}, i}\left(\sum_{j} \psi_{j_{0}}\left(q_{j} y_{j_{1}}\right)\left(\varphi_{i_{0}}\left(v_{i} x_{i_{1}}\right), p_{j}\right)_{B}, f\left(\psi_{j_{1}}\left(\varphi_{i_{1}}\left(a_{1} x_{i_{2}}\right) y_{j_{2}}\right)\right.\right. \\
& \left.\left.\otimes \cdots \otimes \psi_{j_{n}}\left(\varphi_{i_{n}}\left(a_{n} x_{i_{0}}\right) y_{j_{0}}\right)\right)\right)_{C} u_{i} .
\end{aligned}
$$

On the other hand we have that $\operatorname{tr}^{M \otimes_{B} N}(f)$ sends $a_{1} \otimes \cdots \otimes a_{n}$ to

$$
\begin{aligned}
& \sum_{i_{0}, \ldots, i_{n}, j_{0}, \ldots, j_{n}, i}\left(\theta_{i_{0}, j_{0}}\left(v_{i} x_{i_{1}} \otimes y_{j_{1}}\right), f\left(\theta_{i_{1}, j_{1}}\left(a_{1} x_{i_{2}} \otimes y_{j_{2}}\right)\right.\right. \\
& \left.\left.\quad \otimes \cdots \otimes \theta_{i_{n}, j_{n}}\left(a_{n} x_{i_{0}} \otimes y_{j_{0}}\right)\right)\right)_{C} u_{i} \\
& =\sum_{i_{0}, \ldots, i_{n}, j_{0}, \ldots, j_{n}, i}\left(\psi_{j_{0}}\left(\varphi_{i_{0}}\left(v_{i} x_{i_{1}}\right) y_{j_{1}}\right), f\left(\psi_{j_{1}}\left(\varphi_{i_{1}}\left(a_{1} x_{i_{2}}\right) y_{j_{2}}\right)\right.\right. \\
& \left.\left.\quad \otimes \cdots \otimes \psi_{j_{n}}\left(\varphi_{i_{n}}\left(a_{n} x_{i_{0}}\right) y_{j_{0}}\right)\right)\right)_{C} u_{i} .
\end{aligned}
$$

It remains to prove that

$$
\sum_{j} \psi_{j_{0}}\left(q_{j} y_{j_{1}}\right)\left(\varphi_{i_{0}}\left(v_{i} x_{i_{1}}\right), p_{j}\right)_{B}=\psi_{j_{0}}\left(\varphi_{i_{0}}\left(v_{i} x_{i_{1}}\right) y_{j_{1}}\right),
$$

but this follows from the equality

$$
\varphi_{i_{0}}\left(v_{i} x_{i_{1}}\right)=\sum_{j}\left(\varphi_{i_{0}}\left(v_{i} x_{i_{1}}\right), p_{j}\right)_{B} q_{j} .
$$

(2) By assumption, both $\alpha$ and $\beta$ are split as right $B$-module homomorphisms. So there exist right $B$-homomorphisms $\alpha^{\prime}: M \rightarrow L$ and $\beta^{\prime}: N \rightarrow M$ such that $\alpha^{\prime} \alpha=1_{L}, \beta \beta^{\prime}=1_{N}$ and $\alpha \alpha^{\prime}+\beta^{\prime} \beta=1_{M}$. We choose $x_{i} \in L$ and $\varphi_{i} \in \operatorname{Hom}_{B}(L, B)$ with $1 \leq i \leq s$ such that for any $x \in L, x=\sum_{i} x_{i} \varphi_{i}(x)$. Similarly, we choose $y_{j} \in N$ and $\psi_{j} \in \operatorname{Hom}_{B}(N, B)$ with $1 \leq j \leq t$ such that for any $y \in N, y=\sum_{j} y_{j} \psi_{j}(y)$. We define $z_{l} \in M$ and $\pi_{l} \in \operatorname{Hom}_{B}(M, B)$ as follows:

$$
z_{l}=\left\{\begin{array}{cc}
\alpha\left(x_{l}\right) & 1 \leq l \leq s \\
\beta^{\prime}\left(y_{l-s}\right) & s+1 \leq l \leq s+t,
\end{array} \quad \pi_{l}=\left\{\begin{array}{cc}
\varphi_{l} \alpha^{\prime} & 1 \leq l \leq s \\
\psi_{l-s} \beta & s+1 \leq l \leq s+t .
\end{array}\right.\right.
$$

Then for any $z \in M, \sum_{l} z_{l} \pi_{l}(z)=\sum_{i} \alpha\left(x_{i}\right)\left(\varphi_{i} \alpha^{\prime}\right)(z)+\sum_{j} \beta^{\prime}\left(y_{j}\right)\left(\psi_{j} \beta\right)(z)=$ $\alpha\left(\sum_{i} x_{i} \varphi_{i}\left(\alpha^{\prime}(z)\right)+\beta^{\prime}\left(\sum_{j} y_{j} \psi_{j}(\beta(z))=\alpha\left(\alpha^{\prime}(z)\right)+\beta^{\prime}(\beta(z))=z\right.\right.$. As before, we choose bilinear forms $(,)_{A}$ and $(,)_{B}$, respectively, and let $\left\{u_{i}\right\},\left\{v_{i}\right\}$ be dual bases 
in $A$. To prove the statement, it suffices to prove that, for any $f \in \operatorname{Hom}_{k}\left(B^{\otimes n}, B\right)$, $t r^{L}(f)+t r^{N}(f)=t r^{M}(f)$.

For $f \in \operatorname{Hom}_{k}\left(B^{\otimes n}, B\right)$, the map $\operatorname{tr}^{L}(f) \in \operatorname{Hom}_{k}\left(A^{\otimes n}, A\right)$ sends $a_{1} \otimes \cdots \otimes a_{n}$ to

$$
\sum_{i_{0}, \ldots, i_{n}, i}\left(\varphi_{i_{0}}\left(v_{i} x_{i_{1}}\right), f\left(\varphi_{i_{1}}\left(a_{1} x_{i_{2}}\right) \otimes \cdots \otimes \varphi_{i_{n}}\left(a_{n} x_{i_{0}}\right)\right)\right)_{B} u_{i},
$$

and the map $\operatorname{tr}^{N}(f) \in \operatorname{Hom}_{k}\left(A^{\otimes n}, A\right)$ sends $a_{1} \otimes \cdots \otimes a_{n}$ to

$$
\sum_{j_{0}, \ldots, j_{n}, i}\left(\psi_{j_{0}}\left(v_{i} y_{j_{1}}\right), f\left(\psi_{j_{1}}\left(a_{1} y_{j_{2}}\right) \otimes \cdots \otimes \psi_{j_{n}}\left(a_{n} y_{j_{0}}\right)\right)\right)_{B} u_{i} .
$$

On the other hand $t r^{M}(f) \in \operatorname{Hom}_{k}\left(A^{\otimes n}, A\right)$ sends $a_{1} \otimes \cdots \otimes a_{n}$ to

$$
\sum_{l_{0}, \ldots, l_{n}, i}\left(\pi_{l_{0}}\left(v_{i} z_{l_{1}}\right), f\left(\pi_{l_{1}}\left(a_{1} z_{l_{2}}\right) \otimes \cdots \otimes \pi_{l_{n}}\left(a_{n} z_{l_{0}}\right)\right)\right)_{B} u_{i} .
$$

Observe that if $1 \leq j, k \leq s$, then $\pi_{j}\left(v_{i} z_{k}\right)=\varphi_{j}\left(\alpha^{\prime}\left(v_{i} \alpha\left(x_{k}\right)\right)\right)=\varphi_{j}\left(\alpha^{\prime}\left(\alpha\left(v_{i} x_{k}\right)\right)\right)=$ $\varphi_{j}\left(v_{i} x_{k}\right)$. Similarly, if $s+1 \leq j, k \leq s+t$, then $\pi_{j}\left(v_{i} z_{k}\right)=\psi_{j-s}\left(\beta\left(v_{i} \beta^{\prime}\left(y_{k-s}\right)\right)\right)=$ $\psi_{j-s}\left(v_{i} \beta\left(\beta^{\prime}\left(y_{k-s}\right)\right)\right)=\psi_{j-s}\left(v_{i} y_{k-s}\right)$. However, if $1 \leq k \leq s$ and $s+1 \leq j \leq s+t$, then $\pi_{j}\left(v_{i} z_{k}\right)=\psi_{j-s}\left(\beta\left(v_{i} \alpha\left(v_{i} x_{k}\right)\right)\right)=\psi_{j-s}\left(v_{i} \beta\left(\alpha\left(x_{k}\right)\right)\right)=0$. It follows that each term in (iii) occurs exactly once in $(i)$ or $(i i)$, and conversely, each term in $(i)$ or (ii) is a term in $($ iii $)$. Therefore, $($ iii $)=(i)+(i i)$.

(3) Without loss of generality we can assume that $P$ is an indecomposable projective $A$-B-bimodule. Therefore $P \cong A e \otimes_{k} f B$ for some idempotents $e \in A$ and $f \in B$. It follows that $t^{P}=t^{A e} \circ t^{f B}: H H^{n}(B) \rightarrow H H^{n}(A)$ factors through $H H^{n}(k)$. However, $H H^{n}(k)=0$ for each $n \geq 0$.

(4) For the regular $A$ - $A$-bimodule $A$ we choose $x=1, \varphi=1_{A}$ so that $x \varphi(a)=a$ for any $a \in A$. We fix a bilinear form $(,)_{A}$, and let $\left\{u_{i}\right\},\left\{v_{i}\right\}$ be dual bases in $A$. Then for any $f \in \operatorname{Hom}_{k}\left(A^{\otimes n}, A\right)$, we have that $\operatorname{tr}^{A}(f) \in \operatorname{Hom}_{k}\left(A^{\otimes n}, A\right)$ sends $a_{1} \otimes \cdots \otimes a_{n}$ to

$$
\sum_{j}\left(v_{j}, f\left(a_{1} \otimes \cdots \otimes a_{n}\right)_{A} u_{j}=f\left(a_{1} \otimes \cdots \otimes a_{n}\right) .\right.
$$

Remark 2.8. Given a bounded (cochain) complex $X^{\bullet}$ of $A$-B-bimodules whose terms are finitely generated and projective as left and right modules, one can also define a transfer map $t^{X^{\bullet}}: H H^{n}(B) \rightarrow H H^{n}(A)$ by $t^{X^{\bullet}}:=\sum_{i}(-1)^{i} t^{X^{i}}$. Note that if $Y^{\bullet}$ is another bounded complex of $A$-B-bimodules whose terms are finitely generated and projective as left and right modules such that $X^{\bullet}$ and $Y^{\bullet}$ are quasiisomorphic, then $t^{X^{\bullet}}=t^{Y^{\bullet}}$ (by the same argument as in [4, Section 4]).

Let $A$ be a symmetric algebra. Then there is a non-degenerate bilinear pairing between $H H^{n}(A)$ and $H H_{n}(A)$ for any $n \geq 0$ induced by the following isomorphism of complexes:

$$
\begin{aligned}
\operatorname{Hom}_{k}\left(C_{\bullet}(A), k\right) & =\operatorname{Hom}_{k}\left(A \otimes_{A^{e}} \operatorname{Bar}_{\bullet}(A), k\right) \\
& \cong \operatorname{Hom}_{A^{e}}\left(\operatorname{Bar}_{\bullet}(A), A^{*}\right) \cong \operatorname{Hom}_{A^{e}}\left(\operatorname{Bar}_{\bullet}(A), A\right)=C^{\bullet}(A),
\end{aligned}
$$

where the third isomorphism is induced by the isomorphism of bimodules $A^{*} \cong A$.

Lemma 2.9. Let $f \in C^{n}(A) \cong \operatorname{Hom}_{k}\left(A^{\otimes n}, A\right)$. Then its image in $\operatorname{Hom}_{k}\left(A^{\otimes(n+1)}, k\right)$ under the inverse of the above isomorphism, denoted by $\Phi(f)$,

$$
\text { sends } a_{0} \otimes \cdots \otimes a_{n} \text { for } a_{0}, \cdots, a_{n} \in A \text { to }\left(a_{0}, f\left(a_{1} \otimes \cdots \otimes a_{n}\right)\right)_{A} \text {. }
$$


Proof. For $f \in C^{n}(A) \cong \operatorname{Hom}_{k}\left(A^{\otimes n}, A\right)$, its image in $\operatorname{Hom}_{A^{e}}(\operatorname{Bar} \bullet(A), A)$ sends $a_{0} \otimes \cdots \otimes a_{n} \otimes a_{n+1}$ to $a_{0} f\left(a_{1} \otimes \cdots \otimes a_{n}\right) a_{n+1}$. Its image in $\operatorname{Hom}_{A^{e}}\left(\operatorname{Bar}_{\bullet}(A), A^{*}\right)$ sends $a_{0} \otimes \cdots \otimes a_{n} \otimes a_{n+1}$ to $a_{0} f\left(a_{1} \otimes \cdots \otimes a_{n}\right) a_{n+1} s$, where $s$ is the symmetrizing form over $A$. Its image in $\operatorname{Hom}_{k}\left(A \otimes_{A^{e}} \operatorname{Bar} \bullet(A), k\right)$ sends $a \otimes a_{0} \otimes \cdots \otimes a_{n} \otimes a_{n+1}$ to $\left(a_{0} f\left(a_{1} \otimes \cdots \otimes a_{n}\right) a_{n+1} s\right)(a)$. Finally, its image in $\operatorname{Hom}_{k}\left(A^{\otimes(n+1)}, k\right)$ sends $a_{0} \otimes \cdots \otimes$ $a_{n}$ to $\left(a_{0} f\left(a_{1} \otimes \cdots \otimes a_{n}\right) s\right)(1)=s\left(a_{0} f\left(a_{1} \otimes \cdots \otimes a_{n}\right)\right)=\left(a_{0}, f\left(a_{1} \otimes \cdots \otimes a_{n}\right)\right)_{A}$.

Let $A$ and $B$ be two symmetric algebras and let ${ }_{A} M_{B}$ be a bimodule such that ${ }_{A} M$ and $M_{B}$ are finitely generated and projective. Given such a bimodule $M$, transfer maps are defined in Hochschild homology and also in Hochschild cohomology. It will be crucial to know whether they are compatible via the above duality. This question is answered by the following theorem.

Theorem 2.10. Let $A$ and $B$ be two symmetric algebras and let ${ }_{A} M_{B}$ be a bimodule such that ${ }_{A} M$ and $M_{B}$ are finitely generated and projective. Then there is a commutative diagram

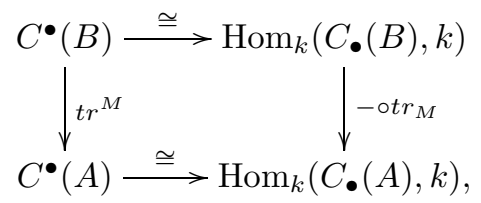

where the horizontal isomorphisms are the above duality and the right-most map is induced by composing $\operatorname{tr}_{M}$ on the right-hand side.

Proof. We compare the action of the two compositions on arbitrary elements.

Let $a_{0}, \ldots, a_{n} \in A$ and let $f \in C^{n}(B)=\operatorname{Hom}_{k}\left(B^{\otimes n}, B\right)$. Then

$$
\begin{aligned}
& \Phi(f) \operatorname{tr}_{M}\left(a_{0} \otimes \cdots \otimes a_{n}\right) \\
= & \Phi(f)\left(\sum_{i_{0}, \ldots, i_{n}} \varphi_{i_{0}}\left(a_{0} x_{i_{1}}\right) \otimes \cdots \otimes \varphi_{i_{n}}\left(a_{n} x_{i_{0}}\right)\right) \\
= & \sum_{i_{0}, \ldots, i_{n}}\left(\varphi_{i_{0}}\left(a_{0} x_{i_{1}}\right), f\left(\varphi_{i_{1}}\left(a_{1} x_{i_{2}}\right) \otimes \cdots \otimes \varphi_{i_{n}}\left(a_{n} x_{i_{0}}\right)\right)\right)_{B},
\end{aligned}
$$

and on the other hand

$$
\begin{aligned}
& \Phi\left(\operatorname{tr}^{M}(f)\right)\left(a_{0} \otimes \cdots \otimes a_{n}\right) \\
= & \left(a_{0}, \operatorname{tr}^{M}(f)\left(a_{1} \otimes \cdots \otimes a_{n}\right)\right)_{A} \\
= & \left(a_{0}, \sum_{i_{0}, \ldots, i_{n}, j}\left(\varphi_{i_{0}}\left(v_{j} x_{i_{1}}\right), f\left(\varphi_{i_{1}}\left(a_{1} x_{i_{2}}\right) \otimes \cdots \otimes \varphi_{i_{n}}\left(a_{n} x_{i_{0}}\right)\right)_{B} u_{j}\right)_{A}\right. \\
= & \sum_{i_{0}, \ldots, i_{n}, j}\left(a_{0}, u_{j}\right)_{A}\left(\varphi_{i_{0}}\left(v_{j} x_{i_{1}}\right), f\left(\varphi_{i_{1}}\left(a_{1} x_{i_{2}}\right) \otimes \cdots \otimes \varphi_{i_{n}}\left(a_{n} x_{i_{0}}\right)\right)_{B}\right. \\
= & \sum_{i_{0}, \ldots, i_{n}}\left(\varphi_{i_{0}}\left(\left(\sum_{j}\left(a_{0}, u_{j}\right)_{A}\right) v_{j} x_{i_{1}}\right), f\left(\varphi_{i_{1}}\left(a_{1} x_{i_{2}}\right) \otimes \cdots \otimes \varphi_{i_{n}}\left(a_{n} x_{i_{0}}\right)\right)_{B}\right. \\
= & \sum_{i_{0}, \ldots, i_{n}}\left(\varphi _ { i _ { 0 } } \left(\left(a_{0} x_{i_{1}}\right), f\left(\varphi_{i_{1}}\left(a_{1} x_{i_{2}}\right) \otimes \cdots \otimes \varphi_{i_{n}}\left(a_{n} x_{i_{0}}\right)\right)_{B},\right.\right.
\end{aligned}
$$

where $\left\{u_{j}\right\},\left\{v_{j}\right\}$ are dual bases in $A$. 
Remark 2.11. An analogous result holds true when replacing the above $M$ by a bounded complex $X^{\bullet}$ of $A$-B-bimodules whose terms are finitely generated and projective as left and right modules.

Corollary 2.12. Let $A$ and $B$ be two symmetric algebras and let ${ }_{A} M_{B}$ be a bimodule such that ${ }_{A} M$ and $M_{B}$ are finitely generated and projective. Then the transfer maps $t^{M}: Z(B) \rightarrow Z(A)$ and $t_{M}: A / K(A) \rightarrow B / K(B)$ satisfy the following compatibility property:

$$
\left(t_{M}(\bar{a}), b\right)_{B}=\left(\bar{a}, t^{M}(b)\right)_{A}, \quad \text { where } \bar{a} \in A / K(A), b \in Z(B) .
$$

Proof. In the degree zero case, both $C_{0}(B)$ and $C^{0}(B)$ identify with $B$ and the duality $\Phi: C^{0}(B) \cong B \rightarrow \operatorname{Hom}_{k}(B, k) \cong \operatorname{Hom}_{k}\left(C_{0}(B), k\right)$ (cf. Lemma 2.9) maps any $b \in B$ to $(-, b)_{B} \in \operatorname{Hom}_{k}(B, k)$. Now the conclusion follows easily from the degree zero case of Theorem 2.10 .

Let $Z^{p r}(A)$ be the projective centre of $A$, that is, the set of $A$ - $A$-bimodule homomorphisms from $A$ to itself which factor through projective bimodules. The projective centre is an ideal of the centre $Z(A)$, and we denote the stable centre by $Z^{s t}(A)=Z(A) / Z^{p r}(A)$.

Lemma 2.13. Let $A$ and $B$ be two symmetric algebras and let ${ }_{A} M_{B}$ be a bimodule such that ${ }_{A} M$ and $M_{B}$ are finitely generated and projective. Then $t^{M}\left(Z^{\text {pr }}(B)\right) \subseteq$ $Z^{p r}(A)$ and hence there is an induced map $t_{s t}^{M}: Z^{s t}(B) \rightarrow Z^{s t}(A)$.

Proof. Note that $t^{M}: Z(B) \rightarrow Z(A)$ coincides with the composition

$$
A \stackrel{\eta_{M^{*}}}{\rightarrow} M \otimes_{B} B \otimes_{B} M^{*} \stackrel{I d_{M} \otimes f \otimes I}{\longrightarrow} d_{M^{*}} M \otimes_{B} B \otimes_{B} M^{*} \stackrel{\varepsilon_{M}}{\longrightarrow} A .
$$

Therefore our conclusion follows from the fact that $M \otimes_{B} P \otimes_{B} M^{*}$ is a projective $A$ - $A$-bimodule for any projective $B$ - $B$-bimodule $P$.

Next we recall the definition of stable Hochschild homology of degree zero and its basic properties (cf. [24]).

Definition 2.14. Let $A$ be a finite dimensional $k$-algebra over a field $k$ with the decomposition ${ }_{A} A=\bigoplus_{i=1}^{r} A e_{i}$, where $A e_{i}(1 \leq i \leq r)$ are indecomposable projective $A$-modules. The stable Hochschild homology group $H H_{0}^{s t}(A)$ of degree zero is defined to be a subgroup of the 0-degree Hochschild homology group $H H_{0}(A)=A / K(A)$, namely

$$
\begin{aligned}
H H_{0}^{\mathrm{st}}(A)=\left\{a \in A \mid \text { the trace of the map } A e_{i} \rightarrow A e_{i}(b \mapsto a b)\right. \\
\text { vanishes for any } 1 \leq i \leq r\} / K(A) .
\end{aligned}
$$

The main property of this group is its invariance under derived equivalences and stable equivalences of Morita type.

Theorem 2.15. (1) [24, Corollary 4.5] Let $A$ and $B$ be two derived equivalent finite dimensional algebras over an algebraically closed field. Then $\operatorname{dim} H H_{0}^{\text {st }}(A)=\operatorname{dim} H H_{0}^{\text {st }}(B)$.

(2) [24, Theorem 4.7] Let $A$ and $B$ be two finite dimensional algebras over an algebraically closed field which are stably equivalent of Morita type. Then $\operatorname{dim} H H_{0}^{\mathrm{st}}(A)=\operatorname{dim} H H_{0}^{\mathrm{st}}(B)$.

(3) [24, Proposition 4.13] If $A$ is symmetric, then $H H_{0}^{s t}(A)=Z^{p r}(A)^{\perp} / K(A)$. 
(4) 24, Proof of Theorem 4.7] Let $M$ be an $A$-B-bimodule such that $M$ induces a stable equivalence of Morita type between $A$ and $B$. Then $t_{M}\left(H H_{0}^{\text {st }}(A)\right) \subseteq$ $H H_{0}^{s t}(B)$ and we denote its restriction on $H H_{0}^{s t}(A)$ by $t_{M}^{s t}: H H_{0}^{s t}(A) \rightarrow$ $H H_{0}^{s t}(B)$.

Now let $A$ and $B$ be two symmetric algebras which are stably equivalent of Morita type (defined by the bimodule ${ }_{A} M_{B}$ ). We have defined two stable transfer maps $t_{s t}^{M}: Z^{s t}(B) \rightarrow Z^{s t}(A)$ and $t_{M}^{s t}: H H_{0}^{s t}(A) \rightarrow H H_{0}^{s t}(B)$. By Theorem 2.15 (3), the symmetrizing form $s \in A^{*}$ induces a non-degenerate bilinear pairing $(,)_{A}: Z^{s t}(A) \times H H_{0}^{s t}(A) \rightarrow k$, and the symmetrizing form $t \in B^{*}$ induces a nondegenerate bilinear pairing $(,)_{B}: Z^{s t}(B) \times H H_{0}^{s t}(B) \rightarrow k$. As in Corollary 2.12, $t_{s t}^{M}$ and $t_{M}^{s t}$ are compatible via $(,)_{A}$ and $(,)_{B}$.

Proposition 2.16. Let $M$ be an $A$-B-bimodule such that $M$ induces a stable equivalence of Morita type between two symmetric algebras $A$ and $B$. Then

$$
\left(t_{M}^{s t}(\bar{a}),[b]\right)_{B}=\left(\bar{a}, t_{s t}^{M}([b])\right)_{A},
$$

where $\bar{a}=a+K(A) \in H H_{0}^{s t}(A)$ and $[b]=b+Z^{p r}(B) \in Z^{s t}(B)$ for $a \in A$ and $b \in Z(B)$.

Proof. This is an easy consequence of Corollary 2.12 and the previous discussion.

\section{TRANSFER MAPS AND PRODUCT STRUCTURE OF HOCHSCHILD COHOMOLOGY}

In this section, we investigate the problem when transfer maps in Hochschild cohomology preserve the product structure of the Hochschild cohomology algebras. We concentrate on the case where the bimodule (bounded complex of bimodules, respectively) is given by a stable equivalence of Morita type (a derived equivalence, respectively).

Recall first the definition of a stable equivalence of Morita type.

Definition 3.1 ([7]). Let $A$ and $B$ be two finite dimensional $k$-algebras. We say that $A$ and $B$ are stably equivalent of Morita type if there are two bimodules ${ }_{A} M_{B}$

and ${ }_{B} N_{A}$ which are projective as left modules and as right modules and such that we have the bimodule isomorphisms

$$
{ }_{A} M \otimes_{B} N_{A} \cong{ }_{A} A_{A} \oplus{ }_{A} P_{A}, \quad{ }_{B} N \otimes_{A} M_{B} \cong{ }_{B} B_{B} \oplus{ }_{B} Q_{B},
$$

where ${ }_{A} P_{A}$ and ${ }_{B} Q_{B}$ are projective bimodules.

From now on, when talking about a stable equivalence of Morita type between algebras $A$ and $B$, we shall always assume that $A$ and $B$ have no semisimple direct summands (as algebras). By [9, Corollary 3.1] and [22, we may and will assume that both $\left(N \otimes_{A}-, M \otimes_{B}-\right)$ and $\left(M \otimes_{B}-, N \otimes_{A}-\right)$ are pairs of adjoint functors. In particular, we can identify $N$ with $\operatorname{Hom}_{A}(M, A)$ as $B$-A-bimodules. The bimodule isomorphism ${ }_{A} M \otimes_{B} N_{A} \cong{ }_{A} A_{A} \oplus{ }_{A} P_{A}$, defines a projection $p: M \otimes_{B} N \rightarrow A$ and an injection $i: A \rightarrow M \otimes_{B} N$. Then we have $p \circ i=1_{A}$. Note that $i$ can be chosen as the unit morphism $\eta_{N}$ of the adjoint pair $\left(N \otimes_{A}-, M \otimes_{B}-\right)$ (see [9]), but in general, for a fixed choice of $i$, one cannot choose $p$ as the counit morphism $\varepsilon_{M}$ of the adjoint pair $\left(M \otimes_{B}-, N \otimes_{A}-\right)$. 
Remark 3.2. Note that in general the composition $\varepsilon_{M} \eta_{N}$ is an invertible element in the centre $Z(A)$. Indeed, $\varepsilon_{M} \eta_{N}$ is an element in the centre $Z(A)$ since both $\varepsilon_{M}$ and $\eta_{N}$ are $A$ - $A$-bimodule homomorphisms. It is also invertible by the following argument. Without loss of generality, we may assume that $A$ is an indecomposable (non-simple) algebra, and therefore the centre $Z(A)$ is a local algebra. Both $\varepsilon_{M}$ and $\eta_{N}$ induce invertible elements in the stable centre $Z^{s t}(A)=Z(A) / Z^{p r}(A)$ and $Z^{p r}(A) \subseteq \operatorname{rad} Z(A)$ in this case. It follows that $\varepsilon_{M} \eta_{N}$ is also invertible in $Z(A)$.

Now suppose that $A$ and $B$ are symmetric $k$-algebras. One can identify $N$ with $M^{*}=\operatorname{Hom}_{k}(M, k)$. Under a suitable choice of symmetrizing forms over $A$ and $B, i$ can be chosen as the unit morphism $\eta_{M^{*}}: A \rightarrow M \otimes_{B} M^{*}$ of the adjoint pair $\left(M^{*} \otimes_{A}-, M \otimes_{B}-\right)$, and $p$ can be chosen as the counit morphism $\varepsilon_{M}: M \otimes_{B} M^{*} \rightarrow A$ of the adjoint pair $\left(M \otimes_{B}-, M^{*} \otimes_{A}-\right)$. Explicit computations for $\eta_{M^{*}}$ and $\varepsilon_{M}$ have been given in Section 2. Note that $\eta_{M^{*}}$ depends on the choice of the symmetrizing form $t$ on $B$ and that $\varepsilon_{M}$ depends on the choice of the symmetrizing form $s$ on $A$. Since the composition $\varepsilon_{M} \eta_{M^{*}}$ is an invertible element, say $u$, in the centre $Z(A)$, we can choose another symmetrizing form $s^{\prime} \in A^{*}$ such that $s^{\prime}=u s$. With respect to $s^{\prime}$ and $t$, the composition $\varepsilon_{M} \eta_{M^{*}}$ is the identity element $1 \in A$.

For a stable equivalence of Morita type, by a result of the second author (22, Corollary 2.4]), if $B$ is symmetric, then so is $A$. More precisely, one can deduce from the bilinear form on $B$ given by $t \in B^{*}$ a bilinear form on $A$ which makes it a symmetric algebra. We shall show that, with respect to this pair of bilinear forms, the composition $\varepsilon_{M} \eta_{M^{*}}$ is the identity element $1 \in A$.

Lemma 3.3. Let $A$ and $B$ be two symmetric algebras which are stably equivalent of Morita type given by ${ }_{A} M_{B}$ and ${ }_{B} N_{A}$. Suppose that $t \in B^{*}$ gives the bilinear form on $B$. Then the induced bilinear form on $A$ can be described as follows:

$$
s: A \rightarrow k, \quad a \mapsto \sum t\left(\varphi_{i}\left(a x_{i}\right)\right),
$$

where $x_{i} \in M$ and $\varphi_{i} \in \operatorname{Hom}_{B}(M, B)$ with $1 \leq i \leq s$ such that for any $x \in M$, $x=\sum_{i} x_{i} \varphi_{i}(x)$.

Proof. The proof follows exactly that of [22, Corollary 2.4]. Since $M_{B}$ is projective, we have by [1, Proposition 20.11] that $M \otimes_{B} D(B) \cong D\left(\operatorname{Hom}_{B}(M, B)\right)$ as $A$ - $B$ bimodules. As ${ }_{B} N$ is projective, by [32, Lemma 3.59], we have $A$ - $A$-bimodule isomorphisms

$$
\begin{aligned}
M \otimes_{B} B \otimes_{B} N & \cong M \otimes_{B} D(B) \otimes_{B} N \\
& \cong D\left(\operatorname{Hom}_{B}(M, B)\right) \otimes_{B} N \\
& \cong D\left(\operatorname{Hom}_{B}\left(N, \operatorname{Hom}_{B}(M, B)\right)\right) \\
& \left.\cong D\left(\operatorname{Hom}_{B}(N, B) \otimes_{B} \operatorname{Hom}_{B}(M, B)\right)\right) \\
& \cong D\left(M \otimes_{B} N\right) .
\end{aligned}
$$

We thus have $M \otimes_{B} N \cong D\left(M \otimes_{B} N\right)$. If we compose it with the injection $j$ : $A \rightarrow M \otimes_{B} N$ and the surjection $D\left(M \otimes_{B} N\right) \rightarrow D(A), f \mapsto f \circ j$, one obtains an $A$ - $A$-bimodule homomorphism

$$
\sigma: A \rightarrow M \otimes_{B} N \cong D\left(M \otimes_{B} N\right) \rightarrow D(A) .
$$

We claim that $\sigma$ is an isomorphism so that $\sigma$ defines a symmetrizing bilinear form for the algebra $A$. In fact, from the isomorphisms $M \otimes_{B} N \cong A \oplus P$ and $M \otimes_{B} N \cong$ 
$D\left(M \otimes_{B} N\right)$, we get an isomorphism $h=\left(\begin{array}{ll}h_{1} & h_{2} \\ h_{3} & h_{4}\end{array}\right): A \oplus P \cong D(A) \oplus D(P)$ with $h_{1}=\sigma$. Suppose that the inverse of $h$ is given by $g=\left(\begin{array}{ll}g_{1} & g_{2} \\ g_{3} & g_{4}\end{array}\right): D(A) \oplus D(P) \cong$ $A \oplus P$. Then we have that $g_{1} h_{1}+g_{2} h_{3}=1_{A} \in \operatorname{End}_{A^{e}}(A)$. Since $A$ has no projective $A^{e}$-summand and $g_{2} h_{3}$ factors through a projective $A^{e}$-module, $g_{2} h_{3} \in$ $\operatorname{radEnd}_{A^{e}}(A)$ and therefore $g_{1} h_{1}=1_{A}-g_{2} h_{3}$ is an isomorphism. It follows that $\sigma=h_{1}$ is an isomorphism.

Now the element $s \in D(A)$ is just the image of $1 \in A$ under $\sigma$ and can be computed explicitly.

Proposition 3.4. Let $A$ and $B$ be two symmetric algebras which are stably equivalent of Morita type given by ${ }_{A} M_{B}$ and ${ }_{B} N_{A}=M^{*}$. Suppose that $t \in B^{*}$ gives the bilinear form over $B$ and that the bilinear form on $A$ is induced from that of $B$ as in Lemma 3.3. Then $\varepsilon_{M} \eta_{M^{*}}=1_{A}$.

Proof. By the explicit computations of $\eta_{M^{*}}$ and $\varepsilon_{M}$ in Section 2 and by Lemma3.3. we have

$$
\varepsilon_{M}\left(\eta_{M^{*}}(1)\right)=\varepsilon_{M}\left(\sum_{i} x_{i} \otimes t \circ \varphi_{i}\right)=\sum_{i j} t \circ \varphi_{i}\left(v_{j} x_{i}\right) u_{j}=\sum_{j} s\left(v_{j}\right) u_{j}=1 .
$$

The conclusion follows from the fact that both $\eta_{M^{*}}$ and $\varepsilon_{M}$ are $A$-A-bimodule homomorphisms.

Now we can state the result that transfer maps preserve product structure once the above choice of bilinear form over $A$ has been made.

Theorem 3.5. Let $A$ and $B$ be two symmetric algebras which are related by a stable equivalence of Morita type that is given by ${ }_{A} M_{B}$ and ${ }_{B} N_{A}=M^{*}$. Suppose that the bilinear form on $A$ is induced from that of $B$ as in Lemma 3.3. Then the transfer map $t^{M}: H H_{s t}^{*}(B)=H H^{*}(B) / Z^{p r}(B) \rightarrow H H_{s t}^{*}(A)$ is an isomorphism of algebras.

This result is indicated without proof in [20, Remark 2.13]. We give a proof here based on a result of Pogorzaly ([27). He proved that a stable equivalence of Morita type between self-injective algebras induces an isomorphism of stable Hochschild cohomology algebras $H H_{s t}^{*}(A)=H H^{*}(A) / Z^{p r}(A)$. He identified $H H_{s t}^{n}(A) \cong$ $\underline{\operatorname{Hom}}_{A^{e}}\left(\Omega_{A^{e}}^{n}(A), A\right)$ and the isomorphism he constructed sends $f: \Omega_{B^{e}}^{n}(B) \rightarrow B$ to the composition in the stable category $A^{e}$-mod,

$$
\Omega_{A^{e}}^{n}(A) \cong M \otimes_{B} \Omega_{B^{e}}^{n}(B) \otimes_{B} N \stackrel{I d_{M} \otimes f \otimes I d_{N}}{\longrightarrow} M \otimes_{B} B \otimes_{B} N \cong A,
$$

where the first isomorphism in the above composition is induced from the injection $i: A \rightarrow M \otimes_{B} N$, and the last isomorphism is induced from the projection $p$ : $M \otimes_{B} N \rightarrow A$. Note that if the algebras are symmetric and ${ }_{B} N_{A}=M^{*}$, then we can choose $i$ as the unit morphism $\eta_{M^{*}}: A \rightarrow M \otimes_{B} M^{*}$ and $p$ as the counit morphism $\varepsilon_{M}: M \otimes_{B} M^{*} \rightarrow A$. To prove Theorem 3.5. it suffices to prove that the above map can be deduced by our transfer map.

Proof. If $g: \operatorname{Bar}_{n}(B) \rightarrow B(n>0)$ is a map of $B^{e}$-modules such that the composition with the differential $d^{\prime}: \operatorname{Bar}_{n+1}(B) \rightarrow \operatorname{Bar}_{n}(B)$ is zero, then we get a map of $B^{e}$-modules $f: \Omega_{B^{e}}^{n}(B) \rightarrow B$; and if $g$ is equal to a composition of a map 
$\operatorname{Bar}_{n-1}(B) \rightarrow B$ with the differential $d^{\prime}: \operatorname{Bar}_{n}(B) \rightarrow \operatorname{Bar}_{n-1}(B)$, then the corresponding $f$ factors through a projective $B^{e}$-module. When $B$ is a self-injective algebra, this gives an isomorphism $H H_{s t}^{n}(B) \cong \underline{\operatorname{Hom}}_{B^{e}}\left(\Omega_{B^{e}}^{n}(B), B\right)(n>0)$. Since $\Theta: \operatorname{Bar}_{\bullet}(A) \rightarrow M \otimes_{B} \operatorname{Bar}_{\bullet}(B) \otimes_{B} N$ is a chain map, we have a commutative diagram

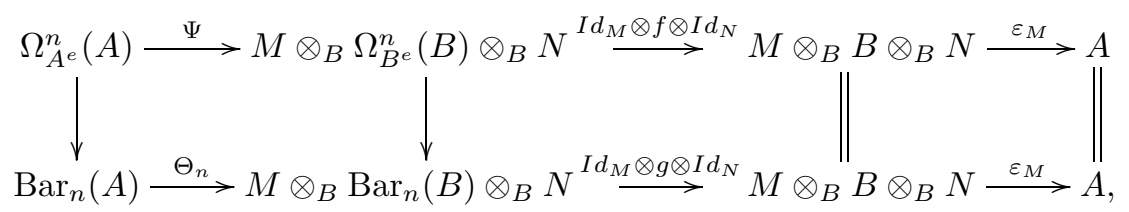

where the first two vertical morphisms are natural injections, where $\Psi$ is induced from $\Theta_{n}$ and thus induced from $\eta_{M^{*}}$. It is easy to see that $\Psi$ is an isomorphism in the stable category of $A^{e}$-modules. Since $\varepsilon_{M}$ is an isomorphism in the stable category, the composition of all morphisms in the upper sequence is exactly the image of $f$ under Pogorzaly's isomorphism. We are done.

Now we look at derived equivalences between symmetric algebras. Let $A$ and $B$ be two finite dimensional symmetric algebras which are derived equivalent given by a two-sided tilting (cochain) complex ${ }_{A} X_{B}^{\bullet}$. Note that ${ }_{A} X_{B}^{\bullet}$ can be chosen as a bounded complex whose terms are finitely generated bimodules and projective as left and right modules. In the following, we always assume that a two-sided tilting complex has this form. It is well known that if $B$ is symmetric, so is $A$. Similarly, we have the following result.

Proposition 3.6. Let $A$ and $B$ be two symmetric algebras, related by a derived equivalence given by a two-sided tilting complex $X^{\bullet}$ whose terms are bimodules that are projective as left $A$-modules and right $B$-modules. Then there is a choice of symmetrizing forms $s$ for $A$ and $t$ for $B$ such that the transfer map $t^{X^{\bullet}}: H H^{*}(B) \rightarrow$ $H H^{*}(A)$ is an isomorphism between Hochschild cohomology algebras.

This result has been indicated without proof in [20, Remark 2.13]. We give a proof here based on the next lemma. Before we state this lemma, we recall the following result proved by Rickard (cf. [17, Theorem 9.2.8]): If $A$ and $B$ are two symmetric algebras related by a derived equivalence given by $X^{\bullet}$, a two-sided tilting complex whose terms are bimodules that are projective as left $A$-modules and right $B$-modules, then $X^{\bullet} \otimes_{B} X^{\bullet *} \cong A$ in the chain homotopy category $K^{b}\left(A \otimes_{k} A^{o p}\right)$ and $X^{\bullet *} \otimes_{A} X^{\bullet} \cong B$ in the chain homotopy category $K^{b}\left(B \otimes_{k} B^{o p}\right)$. Therefore the functors $X^{\bullet} \otimes_{B}-$ and $X^{\bullet *} \otimes_{A}-$ are quasi-inverse equivalences between the chain homotopy categories $K^{b}(A)$ and $K^{b}(B)$, where $X^{\bullet *}$ is the dual complex of $X^{\bullet}$ over $k$. As before, we denote by $\varepsilon_{X} \bullet: X^{\bullet} \otimes_{B} X^{\bullet *} \rightarrow A$ the counit morphism of the adjoint pair $\left(X^{\bullet} \otimes_{B}-, X^{\bullet *} \otimes_{A}-\right)$ and by $\eta_{X} \bullet *: A \rightarrow X^{\bullet} \otimes_{B} X^{\bullet *}$ the unit morphism of the adjoint pair $\left(X^{\bullet *} \otimes_{A}-, X^{\bullet} \otimes_{B}-\right)$.

Lemma 3.7. Let $A$ and $B$ be two symmetric algebras. Fix a symmetrizing form $s$ for $A$ and a symmetrizing form $t$ for $B$. Suppose that $A$ and $B$ are related by $a$ derived equivalence given by a two-sided tilting complex $X^{\bullet}$, whose terms are bimodules that are projective as left $A$-modules and right $B$-modules. Then, in degree zero the counit morphism $\varepsilon_{X} \bullet: X^{\bullet} \otimes_{B} X^{\bullet *} \rightarrow A$ is given by $\varepsilon_{X} \bullet=\sum_{i}(-1)^{i} \varepsilon_{X^{i}}$, and in degree zero the unit morphism $\eta_{X} \bullet *: A \rightarrow X^{\bullet} \otimes_{B} X^{\bullet *}$ is given by $\eta_{X} \bullet *=\sum_{i} \eta_{X^{i *}}$, where $\varepsilon_{X^{i}}$ is the counit morphism of the adjoint pair $\left(X^{i} \otimes_{B}-, X^{i^{*}} \otimes_{A}-\right)$ and 
$\eta_{X^{i *}}$ is the unit morphism of the adjoint pair $\left(X^{i^{*}} \otimes_{A}-, X^{i} \otimes_{B}-\right)$. Moreover, we can choose the symmetrizing form $s$ for $A$ such that the composition $\varepsilon_{X} \bullet \eta_{X} \bullet$ is the identity element in the centre $Z(A)$.

Proof. Given two (cochain) complexes $U^{\bullet}:=\left(\cdots \rightarrow U^{m} \stackrel{d_{U}^{m}}{\rightarrow} U^{m+1} \rightarrow \cdots\right)$ and $V^{\bullet}:=\left(\cdots \rightarrow V^{m} \stackrel{d_{V}^{m}}{\rightarrow} V^{m+1} \rightarrow \cdots\right)$ in $A$-mod, we set the complex

$$
\operatorname{Hom}_{A}^{\bullet}\left(U^{\bullet}, V^{\bullet}\right):=\left(\cdots \rightarrow \operatorname{Hom}_{A}^{m}\left(U^{\bullet}, V^{\bullet}\right) \stackrel{d^{m}}{\rightarrow} \operatorname{Hom}_{A}^{m+1}\left(U^{\bullet}, V^{\bullet}\right) \rightarrow \cdots\right)
$$

where

$$
\begin{aligned}
\operatorname{Hom}_{A}^{m}\left(U^{\bullet}, V^{\bullet}\right) & :=\prod_{j-i=m} \operatorname{Hom}_{A}\left(U^{i}, V^{j}\right) \quad \text { and } \\
d^{m}: \operatorname{Hom}_{A}\left(U^{i}, V^{j}\right) & \rightarrow \operatorname{Hom}_{A}\left(U^{i-1}, V^{j}\right) \times \operatorname{Hom}_{A}\left(U^{i}, V^{j+1}\right), \\
\alpha & \mapsto\left(\alpha d_{U^{\bullet}}^{i-1},(-1)^{i} d_{V}^{j} \alpha\right)
\end{aligned}
$$

In particular, the dual complex of $X^{\bullet}$ over $k$ is given by $X^{\bullet *}$, and $d^{m}:\left(X^{-m}\right)^{*} \rightarrow$ $\left(X^{-m-1}\right)^{*} \quad\left(\alpha \mapsto \alpha d_{U^{\bullet}}^{-m-1}\right)$. Now assume that $W^{\bullet}:=\left(\cdots \rightarrow W^{m} \stackrel{d_{W}^{m}}{\rightarrow} W^{m+1} \rightarrow\right.$ $\cdots)$ is a (cochain) complex in $A^{o p}$-mod. Define the complex

$$
W^{\bullet} \otimes_{A} U^{\bullet}:=\left(\cdots \rightarrow\left(W^{\bullet} \otimes_{A} U^{\bullet}\right)^{m} \stackrel{d^{m}}{\rightarrow}\left(W^{\bullet} \otimes_{A} U^{\bullet}\right)^{m+1} \rightarrow \cdots\right),
$$

where

$$
\begin{gathered}
\left(W^{\bullet} \otimes_{A} U^{\bullet}\right)^{m}:=\bigoplus_{i+j=m} W^{i} \otimes_{A} U^{j} \quad \text { and } \\
d^{m}: W^{i} \otimes_{A} U^{j} \rightarrow\left(W^{i+1} \otimes_{A} U^{j}\right) \oplus\left(W^{i} \otimes_{A} U^{j+1}\right), \\
w \otimes u \mapsto\left(d_{W}^{i} \bullet(w) \otimes u,(-1)^{i} w \otimes d_{U}^{j} \bullet(u)\right) .
\end{gathered}
$$

Now let $X^{\bullet}$ be a two-sided tilting complex whose terms are bimodules that are projective as left $A$-modules and right $B$-modules. Then the adjunction isomorphism $\phi: \operatorname{Hom}_{A}^{\bullet}\left(X^{\bullet} \otimes_{B} Y^{\bullet}, Z^{\bullet}\right) \cong \operatorname{Hom}_{A}^{\bullet}\left(Y^{\bullet}, X^{\bullet *} \otimes_{A} Z^{\bullet}\right)$ can be realized as follows. For any $i, j, k \in \mathbb{Z}$, there is a natural adjunction isomorphism

$$
\phi^{i, j, k}: \operatorname{Hom}_{A}\left(X^{i} \otimes_{B} Y^{j}, Z^{k}\right) \rightarrow \operatorname{Hom}_{A}\left(Y^{j}, X^{i^{*}} \otimes_{A} Z^{k}\right) .
$$

We just put

$$
\phi=\bigoplus_{i, j, k}(-1)^{i j} \phi^{i, j, k} .
$$

From this, the counit morphism $\varepsilon_{X} \bullet$ and the unit morphism $\eta_{X} \bullet *$ can be computed easily. Since the functors $X^{\bullet} \otimes_{B}-$ and $X^{\bullet *} \otimes_{A}-$ are quasi-inverse equivalences, the last statement is clear.

Proof of Proposition 3.6 . We choose the symmetrizing forms $s$ for $A$ and $t$ for $B$ such that the composition $\varepsilon_{X} \bullet \eta_{X} \bullet *$ is the identity element in the centre $Z(A)$. It is well known that derived equivalences preserve the Hochschild cohomology algebras (see, for example, [17, Corollary 6.3.7]). We shall prove that our transfer map coincides with the isomorphism given in [17, Corollary 6.3.7]. First we recall the isomorphism between $H H^{*}(B)$ and $H H^{*}(A)$. Given a representative $f: \operatorname{Bar}_{n}(B) \rightarrow B$ of an element in $H H^{n}(B)$, it corresponds to a morphism $f: \operatorname{Bar}_{\bullet}(B) \rightarrow B[n]$ in 
$K^{b}(B)$. Tensoring from the left by $X^{\bullet}$ and from the right by $X^{\bullet *}$, we get a morphism $I d_{X} \bullet \otimes f \otimes I d_{X} \bullet *: X^{\bullet} \otimes_{B} \operatorname{Bar}_{\bullet}(B) \otimes_{B} X^{\bullet *} \rightarrow X^{\bullet} \otimes_{B} X^{\bullet *}[n]$ in $K^{b}(A)$. Then the composition morphism in $K^{b}(A)$

$$
\begin{aligned}
\left(\varepsilon_{X} \bullet\right. & {[n])\left(I d_{X} \bullet \otimes f \otimes I d_{X} \bullet *\right) \Delta: \operatorname{Bar}_{\bullet}(A) } \\
& \rightarrow X^{\bullet} \otimes_{B} \operatorname{Bar}_{\bullet}(B) \otimes_{B} X^{\bullet *} \rightarrow X^{\bullet} \otimes_{B} X^{\bullet *}[n] \rightarrow A[n]
\end{aligned}
$$

represents the image of $f$ in $H H^{*}(A)$, where $\Delta: \operatorname{Bar} \bullet(A) \rightarrow X^{\bullet} \otimes_{B} \operatorname{Bar} \bullet(B) \otimes_{B} X^{\bullet *}$ lifts the unit morphism $\eta_{X} \bullet *: A \rightarrow X^{\bullet} \otimes_{B} X^{\bullet *}$. Note that $\Delta$ is unique up to homotopy. Since $\eta_{X} \bullet *=\sum_{i} \eta_{X^{i *}}$, we know that $\Delta$ can be taken as $\sum_{i} \Theta_{n, i}$ in degree $-n$, where for each $i$,

$$
\Theta_{n, i}: \operatorname{Bar}_{n}(A) \rightarrow X^{i} \otimes_{B} \operatorname{Bar}_{n}(B) \otimes_{B} X^{i^{*}}
$$

is the morphism which lifts the unit morphism $\eta_{X^{i *}}: A \rightarrow X^{i} \otimes_{B} X^{i^{*}}$ as given in Proposition 2.4. On the other hand, the image of $f$ under our transfer map is given by $t^{X^{\bullet}}(f)=\sum_{i}(-1)^{i} t^{X^{i}}(f)$, where $t^{X^{i}}(f)$ is the following composition (which is the transfer map defined by the $A$ - $B$-bimodule $X^{i}$ ):

$$
\operatorname{Bar}_{n}(A) \stackrel{\Theta_{n, i}}{\rightarrow} X^{i} \otimes_{B} \operatorname{Bar}_{n}(B) \otimes_{B} X^{i^{*} I d_{X^{i}} \otimes f \otimes I d_{X^{i *}}} \stackrel{\longrightarrow}{\longrightarrow} X^{i} \otimes_{B} B \otimes_{B} X^{i^{*} \varepsilon_{X^{i}}} A
$$

It follows that the image of $f$ given in [17, Corollary 6.3.7] coincides with $t^{X^{\bullet}}(f)=$ $\sum_{i}(-1)^{i} t^{X^{i}}(f)$.

\section{P-power maps over the (stable) Hochschild homology OF DEGREE ZERO}

Throughout this section, $k$ denotes a field of characteristic $p>0$. Let $A$ be a finite dimensional $k$-algebra. There exist $p$-power maps over $H H_{0}(A)=A / K(A)$ defined as follows. For any $n \geq 0$,

$$
\mu_{A}^{p^{n}}: H H_{0}(A) \rightarrow H H_{0}(A), \quad a+K(A) \mapsto a^{p^{n}}+K(A) .
$$

These maps are semilinear. Moreover, $\operatorname{Ker}\left(\mu_{A}^{p^{n}}\right)=T_{n}(A) / K(A)$, where

$$
T_{n}(A):=\left\{a \in A \mid a^{p^{n}} \in K(A)\right\},
$$

and $\operatorname{Im}\left(\mu_{A}^{p^{n}}\right)=P_{n}(A) / K(A)$, where

$$
P_{n}(A):=\left\{a^{p^{n}}: a \in A\right\}+K(A) .
$$

Note that if $n=0, T_{0}(A)=K(A)$ and $P_{0}(A)=A$. We thank the referee for pointing out the fact that the abelian group $P_{n}(A)$ is not a vector space unless the ground field $k$ is perfect.

Lemma 4.1 ([24, Lemma 7.1]). Let $A$ and $B$ be two finite dimensional $k$-algebras. Given an $A-B$ bimodule ${ }_{A} M_{B}$, which is finitely generated and projective as a right $B$-module, there is a commutative diagram,

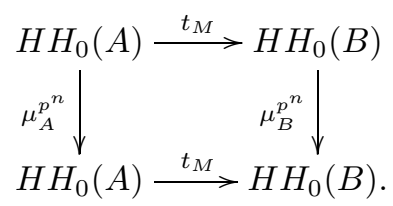


Corollary 4.2. Suppose that two finite dimensional $k$-algebras $A$ and $B$ are related by a derived equivalence that is given by a bounded two-sided tilting complex ${ }_{A} X_{B}^{\bullet}$ whose terms are finitely generated and projective on either side. Then there is a commutative diagram for each $n \geq 0$,

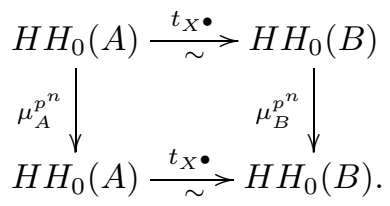

Hence, for each $n \geq 0$,

(1) $\operatorname{dim} T_{n}(A) / K(A)=\operatorname{dim} T_{n}(B) / K(B)$.

(2) If $k$ is a perfect field, then we have $\operatorname{dim} P_{n}(A) / K(A)=\operatorname{dim} P_{n}(B) / K(B)$.

Proof. By Proposition 2.1 and Remark 2.2, the transfer map $t_{X} \bullet: H H_{0}(A) \rightarrow$ $H H_{0}(B)$ is an isomorphism. The commutativity of the diagram follows from the previous lemma, as $t_{X} \bullet=\sum_{i}(-1)^{i} t_{X^{i}}$.

Remark 4.3. The assertion (1) was first proved by C. Bessenrodt, T. Holm and A. Zimmermann ([3, Corollary 1.2]) under the stronger assumption that the ground field $k$ is perfect.

Proposition 4.4. Let $k$ be an algebraically closed field of characteristic $p>0$ and let $A$ be a finite dimensional k-algebra.

(1) For $n \geq 0$, there is an inclusion $\mu_{A}^{p^{n}}\left(H H_{0}^{s t}(A)\right) \subseteq H H_{0}^{s t}(A)$ and thus an induced map

$$
\mu_{A, s t}^{p^{n}}:=\left.\mu_{A}^{p^{n}}\right|_{H H_{0}^{s t}(A)}: H H_{0}^{s t}(A) \rightarrow H H_{0}^{s t}(A) .
$$

(2) For $n \geq 0, \operatorname{Ker}\left(\mu_{A, s t}^{p^{n}}\right)=T_{n}(A) / K(A)$.

(3) For $n \geq 0$, we define $P_{n}^{s t}(A) / K(A):=\operatorname{Im}\left(\mu_{A, s t}^{p^{n}}\right) \subseteq H H_{0}^{s t}(A)$.

(4) $\operatorname{dim} P_{n}(A) / K(A)=\operatorname{dim} P_{n}^{s t}(A) / K(A)+\operatorname{rank}_{p} C_{A}$, where $C_{A}$ is the Cartan matrix of $A$.

Proof. The first assertion is [24, Corollary 7.2]. The second follows from [24, Lemma 7.3] which says that $T_{n}(A) / K(A) \subseteq H H_{0}^{s t}(A)$ for each $n \geq 0$. For the last statement,

$\operatorname{dim} P_{n}(A) / K(A)-\operatorname{dim} P_{n}^{s t}(A) / K(A)=\operatorname{dim} H H_{0}(A)-\operatorname{dim} H H_{0}^{s t}(A)=\operatorname{rank}_{p} C_{A}$,

where the last equality is [24, Theorem 4.4].

Proposition 4.5 ([24, Proposition 7.4]). Let $k$ be an algebraically closed field of characteristic $p>0$ and let $A$ and $B$ be two finite dimensional $k$-algebras. Let ${ }_{A} M_{B}$ be an $A-B$ bimodule, which is finitely generated and projective as right $B$-module. Then we have a commutative diagram,

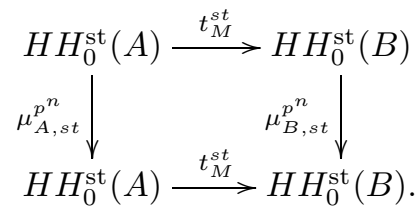


Corollary 4.6. Let $k$ be an algebraically closed field of characteristic $p>0$ and let $A$ and $B$ be two finite dimensional algebras related by a stable equivalence of Morita type that is given by $\left({ }_{A} M_{B},{ }_{B} N_{A}\right)$. Then the following diagram is commutative:

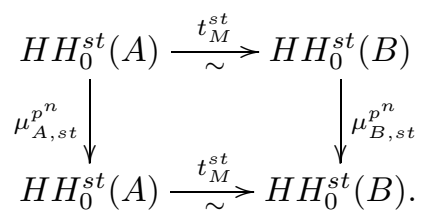

Therefore,

(1) for any $n \geq 0, \operatorname{dim} P_{n}^{s t}(A) / K(A)=\operatorname{dim} P_{n}^{s t}(B) / K(B)$;

(2) for any $n \geq 0, \operatorname{dim} T_{n}(A) / K(A)=\operatorname{dim} T_{n}(B) / K(B)$;

(3) under the condition that $A$ and $B$ have no semisimple summands, the Auslander-Reiten conjecture holds for this stable equivalence of Morita type if and only if $\operatorname{dim} P_{n}(A) / K(A)=\operatorname{dim} P_{n}(B) / K(B)$ for some $n \geq 0$.

Proof. The commutativity of the above diagram was proved in the previous proposition and the assertions (1) and (2) thus follow. The last assertion is implied by (4) of Proposition 4.4 and [24, Proposition 5.1].

Remark 4.7. The maps $\mu_{A, s t}^{p^{n}}$ are invariant under derived equivalences by Proposition 4.5 applied to a two-sided tilting complex.

\section{KÜlShammeR'S MAPS $\zeta_{n}$ AND THEIR STABle VERSiOnS}

Throughout this section, $k$ denotes a perfect field of characteristic $p>0$. Let $A$ be a symmetric $k$-algebra. Then there is a non-degenerate associative symmetric bilinear form $(,)_{A}: A \times A \rightarrow k$. Since $Z(A)=K(A)^{\perp}([19$, (35)]), we have an induced bilinear form, denoted also by $(,)_{A}: Z(A) \times A / K(A) \rightarrow k$. Using the $p$-power maps over $A / K(A)$, for $n \geq 0$, Külshammer introduced a map $\zeta_{n}: Z(A) \rightarrow$ $Z(A)$ by the defining equation

$$
\left(a, a^{p^{n}}+K(A)\right)_{A}=\left(\zeta_{n}(a), a^{\prime}+K(A)\right)_{A}^{p^{n}}
$$

for any $a \in Z(A)$ and $a^{\prime} \in A$. It is easy to see ([19, (46) and (47)]) that $\operatorname{Ker}\left(\zeta_{n}\right)=$ $P_{n}^{\perp}(A)$ and $\operatorname{Im}\left(\zeta_{n}\right)=T_{n}^{\perp}(A)$. One can prove $([19,(36)])$ that $T_{n}^{\perp}(A)$ form a decreasing sequence of ideals in $Z(A)$ and their intersection is $R(A):=Z(A) \cap \operatorname{Soc}(A)$ ([19, (37)]), the so-called Reynolds ideal. The ideals $T_{n}^{\perp}(A)$ are called generalised Reynolds ideals or Külshammer ideals. Note that by [5, Lemma 4.1 (iii)], $Z^{p r}(A) \subseteq R(A) \subseteq T_{n}^{\perp}(A)$ for any $n \geq 0$. (The statement of this result in [5, Lemma 4.1 (iii)] is assuming that $k$ is algebraically closed, but it is easy to verify that the proof doesn't use this assumption.)

Proposition 5.1. Let $A$ and $B$ be two symmetric $k$-algebras and let ${ }_{A} M_{B}$ be a bimodule which is finitely generated and projective on either side. Then the following diagram is commutative:

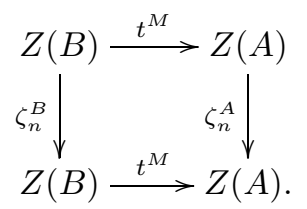


Proof. For any $a \in A$ and $b \in Z(B)$, write $\bar{a}=a+K(A) \in A / K(A)$. Then

$$
\begin{aligned}
\left(t^{M}\left(\zeta_{n}^{B}(b)\right), \bar{a}\right)_{A}^{p^{n}} & =\left(\zeta_{n}^{B}(b), t_{M}(\bar{a})\right)_{B}^{p^{n}} \\
& =\left(b, t_{M}(\bar{a})^{p^{n}}\right)_{B} \\
& =\left(b, t_{M}\left(\bar{a}^{p^{n}}\right)\right)_{B} \\
& =\left(t^{M}(b), \bar{a}^{p^{n}}\right)_{A} \\
& =\left(\zeta_{n}^{A}\left(t^{M}(b)\right), \bar{a}\right)_{A}^{p^{n}},
\end{aligned}
$$

where the first and the fourth equality use Corollary 2.12 and the third one uses Lemma 4.1. Since $a$ is arbitrary, this implies $t^{M}\left(\zeta_{n}^{B}(b)\right)=\zeta_{n}^{A}\left(t^{M}(b)\right)$.

Now we can give a new proof of the following theorem of Zimmermann.

Theorem 5.2 ([35, Theorem 1]). Let $A$ and $B$ be symmetric $k$-algebras. Let $A$ and $B$ be derived equivalent given by a bounded two-sided tilting complex $X{ }^{\bullet}$ whose terms are finitely generated and projective on either side. Then symmetrizing forms $s$ for $A$ and $t$ for $B$ can be chosen in such a way that the following diagram is commutative:

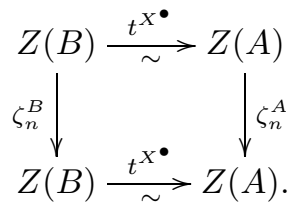

Thus

(1) for $n \geq 0, t^{X^{\bullet}}\left(T_{n}^{\perp}(B)\right)=T_{n}^{\perp}(A)$, that is, $T_{n}^{\perp}(B)$ and $T_{n}^{\perp}(A)$ are isomorphic as ideals via the algebra isomorphism $t^{X^{\bullet}}: Z(B) \rightarrow Z(A)$;

(2) for $n \geq 0, t^{X^{\bullet}}\left(P_{n}^{\perp}(B)\right)=P_{n}^{\perp}(A)$.

Proof. The isomorphism is a consequence of Proposition 3.6. Since $t^{X^{\bullet}}=$ $\sum_{i}(-1)^{i} t^{X^{i}}$, the commutativity of the diagram follows from the preceding proposition.

Now we consider the stable version of $\zeta_{n}^{A}$.

Lemma 5.3. (1) Let $A$ be a finite dimensional algebra. Fix $n \geq 0$. If $a+$ $K(A) \in H H_{0}^{s t}(A) \cap\left(P_{n}(A) / K(A)\right)$, then there exists $b+K(A) \in H H_{0}^{s t}(A)$ such that $a-b^{p} \in K(A)$.

(2) Let $A$ be a symmetric algebra. Then the inverse image of $Z^{p r}(A)$ under $\zeta_{n}^{A}: Z(A) \rightarrow Z(A)$ is $P_{n}^{\perp}(A)+Z^{p r}(A)$

Proof. (1) Let $\left\{e_{1}, \ldots, e_{n}\right\}$ be a complete list of representatives of primitive orthogonal idempotents. Let $t_{A e_{i}}: H H_{0}(A) \rightarrow H H_{0}(k)=k$ be the transfer maps in Hochschild homology. If $a+K(A) \in H H_{0}^{s t}(A) \cap P_{n}(A) / K(A)$, then there exists $b+K(A) \in H H_{0}(A)$ such that $a-b^{p^{n}} \in K(A)$ and $t_{A e_{i}}\left(b^{p^{n}}+K(A)\right)=0$ for all $i$. By [24, Lemma 7.1], $t_{A e_{i}}(b+K(A))^{p^{n}}=0$ for all $i$, and therefore $t_{A e_{i}}(b+K(A))=0$ for all $i$ since $k$ is perfect. This implies $b+K(A) \in H H_{0}^{s t}(A)$.

(2) Let $a \in Z(A)$. Then $\zeta_{n}(a) \in Z^{p r}(A)$ if and only if $a$ is orthogonal to the subspace generated by $\left\{b^{p^{n}}+K(A) \in H H_{0}(A): b+K(A) \in H H_{0}^{\text {st }}(A)=\right.$ $\left.Z^{p r}(A)^{\perp} / K(A)\right\}$. By (1), this subspace is just $\left(Z^{p r}(A)^{\perp} \cap P_{n}(A)\right) / K(A)$ and thus $a \in P_{n}^{\perp}(A)+Z^{p r}(A)$. 
Proposition 5.4. Suppose that $A$ is a symmetric k-algebra.

(1) For $n \geq 0$, we have $\zeta_{n}^{A}\left(Z^{p r}(A)\right) \subseteq Z^{p r}(A)$ and thus an induced map: $\zeta_{n}^{A, s t}$ : $Z^{\text {st }}(A) \rightarrow Z^{\text {st }}(A)$.

(2) For $n \geq 0, \operatorname{Im}\left(\zeta_{n}^{A, s t}\right)=T_{n}^{\perp}(A) / Z^{p r}(A)$.

(3) For $n \geq 0, \operatorname{Ker}\left(\zeta_{n}^{A, s t}\right) \simeq P_{n}^{\perp}(A)$.

Proof. Let $a^{\prime}+K(A) \in H H_{0}^{s t}(A)$. Then $a^{\prime p^{n}}+K(A) \in H H_{0}^{s t}(A)=Z^{p r}(A)^{\perp} / K(A)$ by Proposition 4.4(1). For $a \in Z^{p r}(A),\left(\zeta_{n}(a), a^{\prime}+K(A)\right)=\left(a,\left(a^{\prime}\right)^{p^{n}}+K(A)\right)^{p^{-n}}=$ 0 and (1) follows.

The assertion (2) is obvious, since $Z^{p r}(A) \subseteq T_{n}^{\perp}(A)$ for any $n \geq 0$.

By (2), we have

$$
\operatorname{dimKer}\left(\zeta_{n}^{A, s t}\right)=\operatorname{dim} Z^{s t}(A)-\operatorname{dimIm}\left(\zeta_{n}^{A, s t}\right)=\operatorname{dim} Z(A) / T_{n}^{\perp}(A)=\operatorname{dim} P_{n}^{\perp}(A) .
$$

On the other hand, by Lemma 5.3 (2), we have

$$
\operatorname{Ker}\left(\zeta_{n}^{A, s t}\right)=\left(P_{n}^{\perp}(A)+Z^{p r}(A)\right) / Z^{p r}(A) \cong P_{n}^{\perp}(A) /\left(P_{n}^{\perp}(A) \cap Z^{p r}(A)\right) .
$$

So $Z^{p r}(A) \cap P_{n}^{\perp}(A)=\{0\}$ and (3) follows.

Remark 5.5. The above proof shows that for a symmetric algebra $A$ defined over a perfect field of characteristic $p>0, Z^{p r}(A) \cap P_{n}^{\perp}(A)=\{0\}$ for $n \geq 0$.

Remark 5.6. Let $A$ be a symmetric algebra. Since $H H_{0}^{s t}(A)=Z^{p r}(A)^{\perp} / K(A)$, we have an induced non-degenerate bilinear pairing $Z^{s t}(A) \times H H_{0}^{s t}(A) \rightarrow k$. The map $\zeta_{n}^{A, s t}$ satisfies

$$
\left(\zeta_{n}^{A, s t}([a]), \overline{a^{\prime}}\right)^{p^{n}}=\left([a], \mu_{A}^{p^{n}}\left(\overline{a^{\prime}}\right)\right),
$$

where $[a]=a+Z^{p r}(A) \in Z^{s t}(A)$ and $\overline{a^{\prime}}=a^{\prime}+K(A) \in H H_{0}^{s t}(A)$ for $a \in Z(A)$ and $a^{\prime} \in A$. This equation can also be used to define $\zeta_{n}^{A, s t}$.

Corollary 5.7. Let $A$ and $B$ be two symmetric $k$-algebras and let ${ }_{A} M_{B}$ be a bimodule which is finitely generated and projective on either side. Then there is a commutative diagram,

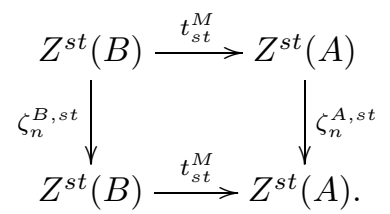

Proof. This follows from Proposition 5.1 and Proposition 5.4(1).

Proposition 5.8. Let $A$ and $B$ be two finite dimensional symmetric algebras which are related by a stable equivalence of Morita type given by $\left({ }_{A} M_{B},{ }_{B} N_{A}\right)$. Suppose that the bilinear form of $A$ is induced from that of $B$. Then there is a commutative diagram,

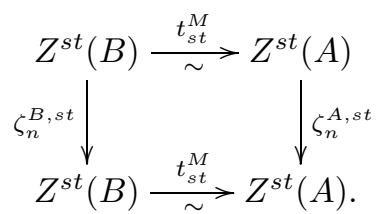


Thus

(1) for $n \geq 0, \operatorname{dim} P_{n}^{\perp}(A)=\operatorname{dim} P_{n}^{\perp}(B)$;

(2) for $n \geq 0, T_{n}^{\perp}(B) / Z^{p r}(B)$ and $T_{n}^{\perp}(A) / Z^{p r}(A)$ are isomorphic as ideals via the algebra isomorphism $t_{s t}^{M}: Z^{s t}(B) \rightarrow Z^{\text {st }}(A)$;

(3) under the condition that $A$ and $B$ have no semisimple summands, the Auslander-Reiten conjecture holds for this stable equivalence of Morita type if and only if $\operatorname{dim} T_{n}^{\perp}(A)=\operatorname{dim} T_{n}^{\perp}(B)$ for some $n \geq 0$.

Proof. By [22, $B$ is also symmetric. The proof easily follows from Theorem 3.5 and Corollary 5.7 .

Remark 5.9. (1) The above claim (2) generalises [24, Proposition 7.10] which proved an equality of dimensions

$$
\operatorname{dim} T_{n}^{\perp}(B) / Z^{p r}(B)=\operatorname{dim} T_{n}^{\perp}(A) / Z^{p r}(A) .
$$

(2) For $n=0, T_{0}^{\perp}(A)=Z(A)$. Thus claim (3) generalises [24, Corollary 1.2] in case of positive characteristic.

(3) The stable version $\zeta_{n}^{s t}$ is invariant also under derived equivalences, by Corollary 5.7.

\section{P-power maps over the (stable) Centre AND KÜLSHAMMER'S MAPS $\kappa_{n}$}

Throughout this section, $k$ denotes a perfect field of characteristic $p>0$. Let $A$ be a symmetric $k$-algebra. In [18, using the $p$-power maps over $Z(A)$, for $n \geq 0$, Külshammer introduced a map $\kappa_{n}^{A}: H H_{0}(A) \rightarrow H H_{0}(A)$ by the defining equation

$$
\left(a^{p^{n}}, \overline{a^{\prime}}\right)=\left(a, \kappa_{n}^{A}\left(\overline{a^{\prime}}\right)\right)^{p^{n}}
$$

for any $a \in Z(A)$ and $a^{\prime} \in A$. This construction is in some sense dual to that of the maps $\zeta_{n}$ and we can also define a stable version of $\kappa_{n}^{A}$. In contrast to the case of $\zeta_{n}$, however, we cannot give a satisfactory description of their kernels and cokernels.

Let $A$ be a finite dimensional $k$-algebra. Since the centre $Z(A)$ is a commutative algebra, the $p$-power maps preserve the multiplication in $Z(A)$ and they are denoted by $\mu_{p^{n}}^{A}: Z(A) \rightarrow Z(A), a \mapsto a^{p^{n}}$ for $n \geq 0$. Note that $\operatorname{Ker} \mu_{p^{n}}^{A}=T_{n}(Z(A)):=$ $\left\{a \in Z(A): a^{p^{n}}=0\right\}$ and $\operatorname{Im} \mu_{p^{n}}^{A}=P_{n}(Z(A)):=\left\{a^{p^{n}}: a \in Z(A)\right\}$. Since derived equivalences preserve the centres as algebras, these maps are invariant under derived equivalences. Since $Z^{p r}(A)$ is an ideal of $Z(A), \mu_{p^{n}}^{A}\left(Z^{p r}(A)\right) \subseteq Z^{p r}(A)$. So there is an induced map $\mu_{p^{n}}^{A, s t}: Z^{s t}(A) \rightarrow Z^{s t}(A)$. Note that if $n=0$, then $T_{0}(Z(A))=0$ and $P_{0}(Z(A))=Z(A)$. Proposition 3.5 gives the commutative diagram,

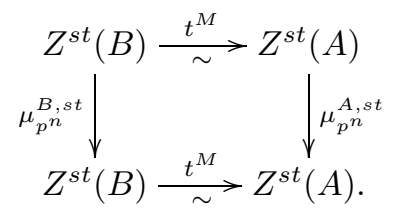

Lemma 6.1. Let $A$ be a Frobenius algebra. Then $Z^{p r}(A) \subseteq \operatorname{Soc}(A)$. As a consequence, if $A$ has no semisimple summands, then $Z^{p r}(A)^{2}=0$ and $Z^{p r}(A) \subseteq$ $T_{n}(Z(A))$ for $n \geq 1$. 
Proof. The proof imitates that of [24, Proposition 4.11]. Let $\left\{a_{i}\right\}$ be a basis of $A$ and let $\left\{b_{i}\right\}$ be a dual basis with respect to the bilinear form over $A$, that is, $\left(a_{i}, b_{j}\right)=\delta_{i j}$. Then by [24, Proposition 1.3 (1)], $Z^{p r}(A)$ is the image of the map $\tau: A \rightarrow A, x \mapsto \sum b_{i} x a_{i}$. We shall prove that $\operatorname{Im}(\tau) \subseteq \operatorname{Soc}(A)$.

We carefully choose a basis $\left\{a_{i}\right\}$ and its dual basis $\left\{b_{i}\right\}$ in $A$, as follows. Suppose that

$$
A / J(A) \simeq M_{u_{1}}(k) \times \cdots \times M_{u_{r}}(k) .
$$

Write $E_{i j}^{t}$ the matrix in $M_{u_{t}}(k)$ whose entry at the position $(i, j)$ is 1 and is zero elsewhere. Then take $a_{1}=e_{1}, a_{2}=e_{2}, \ldots, a_{m}=e_{m} \in A$ such that their images in $A / J(A)$ correspond to the matrices $E_{i i}^{t}$ for $1 \leq i \leq u_{t}$ and $1 \leq t \leq r$ and take $a_{m+1}, \ldots, a_{n}$ such that their images in $A / J(A)$ correspond to $E_{i j}^{t}$ for $1 \leq i \neq j \leq u_{t}$ and $1 \leq t \leq r$. Then $\left\{a_{1}, \ldots, a_{n}\right\}$ are linearly independent in $A$ and their images in $A / J(A)$ form a basis of the vector space $A / J(A)$. Note that for $m+1 \leq u \leq n$, $a_{u} \in e_{i} A e_{j}$ for some $1 \leq i \neq j \leq m$. Moreover, let $a_{n+1}, \ldots, a_{s} \in J(A)$ such that their images in $J(A) / J^{2}(A)$ are a basis of the vector space $J(A) / J^{2}(A)$, let $a_{s+1}, \ldots, a_{t} \in J^{2}(A)$ such that their images in $J^{2}(A) / J^{3}(A)$ are a basis of the vector space $J^{2}(A) / J^{3}(A)$, and so on. Let $b_{1}, b_{2}, \ldots$ be the dual basis. Then $\left\{b_{1}, \ldots, b_{n}\right\}$ is a basis of $J(A)^{\perp}=\operatorname{Soc}(A),\left\{b_{1}, \ldots, b_{s}\right\}$ is a basis of $J^{2}(A)^{\perp},\left\{b_{1}, \ldots, b_{t}\right\}$ is a basis of $J^{3}(A)^{\perp}$, etc. Now we can prove the first assertion. Since for any $x \in A$,

$$
J(A) x=0 \Longleftrightarrow x \in \operatorname{Soc}(A) \Longleftrightarrow x J(A)=0,
$$

we need to prove that $J(A) \cdot \operatorname{Im}(\tau)=0$. It is easy to see that $J(A) \cdot J^{n}(A)^{\perp}$. $J^{n-1}(A)=0$ for $n \geq 1$. Let $y \in J(A)$ and $x \in A$. For $1 \leq i \leq n$, we get $y b_{i} x a_{i} \in$ $J(A) \cdot \operatorname{Soc}(A) \cdot A \cdot A=0$; for $n+1 \leq i \leq s$, we get $y b_{i} x a_{i} \in J(A) \cdot J^{2}(A)^{\perp} \cdot A \cdot J(A)=0$; for $s+1 \leq i \leq t$, we get $y a_{i} x b_{i} \in J(A) \cdot J^{3}(A)^{\perp} \cdot A \cdot J^{2}(A)=0$, etc. This proves that $\operatorname{Im}(\tau) \subseteq \operatorname{Soc}(A)$.

Now if $A$ has no semisimple direct summand, then $Z^{p r}(A)^{2} \subseteq(\operatorname{Soc}(A))^{2} \subseteq$ $\operatorname{Soc}(A) J(A)=0$ and thus for $n \geq 1, Z^{p r}(A) \subseteq T_{n}(Z(A))$.

As a consequence, we have

Corollary 6.2. Let $A$ be a finite dimensional algebra without semisimple direct summands. Then for $n \geq 1$,

(1) $T_{n}(Z(A)) / Z^{p r}(A) \subseteq \operatorname{Ker} \mu_{p^{n}}^{A, s t} \subseteq T_{n+1}(Z(A)) / Z^{p r}(A)$;

(2) $P_{n}(Z(A)) \rightarrow \operatorname{Im} \mu_{p^{n}}^{A, s t} \rightarrow P_{n+1}(Z(A))$.

The inclusions in (1) can be strict, as illustrated by the following

Example 6.3. Let $A=k[X] /\left(X^{p^{m}+1}\right)$ with $m \geq 1$. Then $Z^{p r}(A)=\left(X^{p^{m}}\right) /\left(X^{p^{m}+1}\right)$ and $Z^{s t}(A)=Z(A) / Z^{p r}(A) \cong k[X] /\left(X^{p^{m}}\right)$. Hence, for $1 \leq n \leq m, T_{n}(Z(A)) / Z^{p r}(A)$ $=\left\langle\overline{X^{i}}, p^{m-n}<i \leq p^{m}-1\right\rangle$ and $\operatorname{Ker} \mu_{p^{n}}^{A, s t}=\left\langle\overline{X^{i}}, p^{m-n} \leq i \leq p^{m}-1\right\rangle$.

Now we consider the stable version of $\kappa_{n}$. Recall that $\operatorname{Ker}\left(\kappa_{n}^{A}\right)=P_{n}^{\perp}(Z(A)) / K(A)$ and $\operatorname{Im}\left(\kappa_{n}^{A}\right)=T_{n}^{\perp}(Z(A)) / K(A)([19$, (52)(53)]).

Proposition 6.4 ([36, Proposition 2.3]). Let $A$ and $B$ be symmetric k-algebras. Suppose that $A$ and $B$ are derived equivalent, and the equivalence is given by a bounded two-sided tilting complex $X^{\bullet}$ whose terms are finitely generated and projective on either side. Then there exists an isomorphism $t_{X} \bullet: H H_{0}(A) \stackrel{\sim}{\rightarrow} H H_{0}(B)$ 
such that

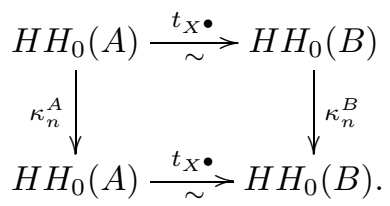

Proof. For any $b \in Z(B)$ and $a \in A$, write $\bar{a}=a+K(A) \in H H_{0}(A)$. We have

$$
\begin{aligned}
\left(b, \kappa_{n}^{B} t_{X} \cdot(\bar{a})\right)_{B}^{p^{n}} & =\left(b^{p^{n}}, t_{X} \cdot(\bar{a})\right)_{B} \\
& =\left(t^{X^{\bullet}}\left(b^{p^{n}}\right), \bar{a}\right)_{A} \\
& =\left(t^{X^{\bullet}}(b)^{p^{n}}, \bar{a}\right)_{A} \\
& =\left(t^{X^{\bullet}}(b), \kappa_{n}^{A}(\bar{a})\right)_{A}^{p^{n}} \\
& =\left(b, t_{X} \cdot \kappa_{n}^{A}(\bar{a})\right)_{B}^{p^{n}},
\end{aligned}
$$

where the second and the fifth equality use Corollary 2.12 and the third one uses Proposition 3.6. We thus have $\kappa_{n}^{B} t_{X} \bullet(\bar{a})=t_{X} \bullet \kappa_{n}^{A}(\bar{a})$, as $b \in Z(B)$ is arbitrary.

Now we consider the stable version of $\kappa_{n}^{A}$.

Lemma 6.5. Suppose that $A$ is a symmetric algebra. For $n \geq 0$, we have $\kappa_{n}\left(H H_{0}^{s t}(A)\right) \subseteq H H_{0}^{s t}(A)$ and thus an induced map $\kappa_{n}^{A, s t}: H H_{0}^{s t}(A) \rightarrow H H_{0}^{s t}(A)$.

Proof. For $\overline{a^{\prime}}=a^{\prime}+K(A) \in H H_{0}^{s t}(A)=Z^{p r}(A)^{\perp} / K(A)$ and $a \in Z^{p r}(A)$, since $a^{p^{n}} \in Z^{p r}(A)$, we have

$$
\left(a, \kappa_{n}^{A}\left(\overline{a^{\prime}}\right)\right)_{A}^{p^{n}}=\left(a^{p^{n}}, \overline{a^{\prime}}\right)_{A}=0 .
$$

Thus $\kappa_{n}^{A}\left(\overline{a^{\prime}}\right) \in H H_{0}^{s t}(A)$.

Remark 6.6. Let $A$ be a symmetric algebra. Since we have an induced nondegenerate bilinear pairing $Z^{s t}(A) \times H H_{0}^{s t}(A) \rightarrow k$, the map $\kappa_{n}^{A, s t}$ satisfies

$$
\left([a], \kappa_{n}^{A, s t}\left(\overline{a^{\prime}}\right)\right)^{p^{n}}=\left([a]^{p^{n}}, \overline{a^{\prime}}\right),
$$

where $[a]=a+Z^{p r}(A) \in Z^{s t}(A)$ and $\overline{a^{\prime}}=a^{\prime}+K(A) \in H H_{0}^{s t}(A)$ for $a \in Z(A)$ and $a^{\prime} \in A$. This equation can also be used to define $\kappa_{n}^{A, s t}$.

Although we don't have a good description of the kernel and the cokernel of $\kappa_{n}^{A, s t}$, we record the following

Proposition 6.7. Let $A$ and $B$ be two finite dimensional symmetric algebras which are stably equivalent of Morita type, where the equivalence is given by $\left({ }_{A} M_{B},{ }_{B} N_{A}\right)$. Suppose that the bilinear form of $A$ is induced from that of $B$. Then the following diagram is commutative:

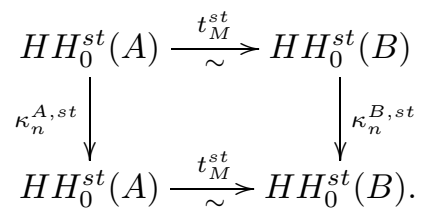

Proof. This is obvious.

Remark 6.8. The new maps $\kappa_{n}^{s t}$ are invariant under derived equivalences between symmetric algebras, by an analogue of Proposition 6.7. 


\section{Higher Dimensional anAlOgUe}

Zimmermann introduced higher dimensional analogues of the map $\kappa_{n}$ in [36]. Let us recall his construction.

Let $A$ be a symmetric algebra defined over a perfect field of characteristic $p>0$. We have a non-degenerate bilinear paring (cf. Lemma 2.9) for each $m \geq 0$,

$$
(,)_{m}: H H^{m}(A) \times H H_{m}(A) \rightarrow k,
$$

which generalizes the form in degree zero $():, Z(A) \times A / K(A) \rightarrow k$. Since there exists a cup product on the Hochschild cohomology, one defines $\kappa_{n}^{(m), A}$ : $H H_{p^{n} m}(A) \rightarrow H H_{m}(A)$ by the equation

$$
\left(f^{p^{n}}, x\right)_{p^{n} m}=\left(f, \kappa_{n}^{(m)}(x)\right)_{m}^{p^{n}}
$$

for $f \in H H^{m}(A)$ and $x \in H H_{p^{n} m}(A)$. Obviously, $\kappa_{n}^{(0), A}=\kappa_{n}^{A}$.

Theorem 7.1. Let $A$ and $B$ be two symmetric $k$-algebras

(1) (36, Theorem 1]) Suppose that $A$ and $B$ are related by a derived equivalence that is given by a bounded two-sided tilting complex ${ }_{A} X_{B}^{\bullet}$ whose terms are finitely generated and projective on either side. Then for each $m \geq 1$, there is a commutative diagram,

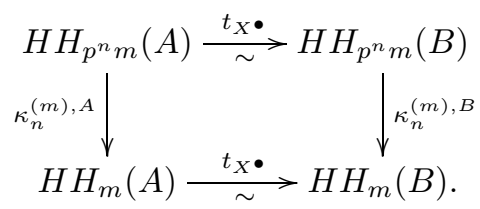

(2) Suppose that $A$ and $B$ are related by a stable equivalence of Morita type that is given by $\left({ }_{A} M_{B},{ }_{B} N_{A}\right)$. Then for each $m \geq 1$, there is a commutative diagram,

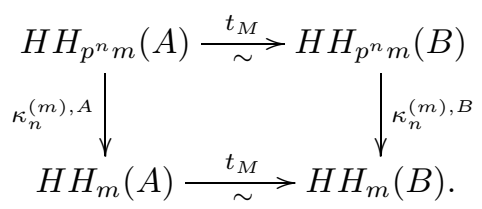

Proof. The proof can be obtained by imitating the proofs of Propositions 6.4 and 6.7 using Proposition 3.6 or Theorem 3.5.

Remark 7.2. One can also obtain a proof of (2) in the above result by imitating the original proof of Zimmermann.

\section{TRIVIAL EXTENSIONS}

Let $A$ be a finite dimensional algebra defined over a perfect field $k$ of characteristic $p>0$. We denote by $A^{*}$ the $k$-linear dual $\operatorname{Hom}_{k}(A, k)$ which becomes an $A$ - $A$-bimodule by setting $\left(a f a^{\prime}\right)(b)=f\left(a^{\prime} b a\right)$ for all $a, a^{\prime}, b \in A$ and $f \in A^{*}$.

The trivial extension $\mathbb{T}(A):=A \oplus A^{*}$ is the $k$-algebra defined by the multiplication $(a, f)(b, g):=(a b, a g+f b)$ for all $a, b \in A$ and $f, g \in A^{*}$. The trivial extension $\mathbb{T}(A)$ is a symmetric algebra, with respect to the bilinear form $((a, f),(b, g))=$ $f(b)+g(a)$. 
Let $\left[A, A^{*}\right]$ denote the commutator subspace of $A^{*}$, that is, the subspace generated by $a f-f a$ for arbitrary $a \in A$ and $f \in A^{*}$. If $V$ is a subspace of $A$, then $\operatorname{Ann}_{A^{*}}(V):=\left\{f \in A^{*} \mid f(V)=0\right\}$.

The following long proposition is collecting properties of the trivial extension algebra.

Proposition 8.1. (1) $Z(\mathbb{T}(A))=Z(A) \oplus \operatorname{Ann}_{A^{*}}(K(A))$;

(2) $K(\mathbb{T}(A))=K(A) \oplus\left[A, A^{*}\right]$;

(3) $\mathbb{T}(A) / K(\mathbb{T}(A))=A / K(A) \oplus A^{*} /\left[A, A^{*}\right]$;

(4) for $n \geq 1, T_{n}(\mathbb{T}(A))=T_{n}(A) \oplus A^{*}$;

(5) for $n \geq 1, P_{n}(\mathbb{T}(A))=P_{n}(A) \oplus\left[A, A^{*}\right]$;

(6) for $n \geq 1, T_{n}(\mathbb{T}(A)) / K(\mathbb{T}(A))=T_{n}(A) / K(A) \oplus A^{*} /\left[A, A^{*}\right]$;

(7) for $n \geq 1, P_{n}(\mathbb{T}(A)) / K(\mathbb{T}(A))=P_{n}(A) / K(A) \oplus 0$;

(8) for $n \geq 1, T_{n}^{\perp}(\mathbb{T}(A))=0 \oplus \operatorname{Ann}_{A^{*}}\left(T_{n}(A)\right)$;

(9) for $n \geq 1, P_{n}^{\perp}(\mathbb{T}(A))=Z(A) \oplus \operatorname{Ann}_{A^{*}}\left(P_{n}(A)\right)$;

(10) for $n \geq 1, T_{n}(Z(\mathbb{T}(A)))=T_{n}(Z(A)) \oplus \operatorname{Ann}_{A^{*}}(K(A))$;

(11) for $n \geq 1, P_{n}(Z(\mathbb{T}(A)))=P_{n}(Z(A)) \oplus 0$;

(12) for $n \geq 1, T_{n}^{\perp}(Z(\mathbb{T}(A)))=K(A) \oplus \operatorname{Ann}_{A^{*}}\left(T_{n}(Z(A))\right)$;

(13) for $n \geq 1, P_{n}^{\perp}(Z(\mathbb{T}(A)))=A \oplus \operatorname{Ann}_{A^{*}}\left(P_{n}(Z(A))\right)$;

(14) for $n \geq 1, T_{n}^{\perp}(Z(\mathbb{T}(A))) / K(\mathbb{T}(A))=0 \oplus \operatorname{Ann}_{A^{*}}\left(T_{n}(Z(A))\right) /\left[A, A^{*}\right]$;

(15) for $n \geq 1, P_{n}^{\perp}(Z(\mathbb{T}(A))) / K(\mathbb{T}(A))=A / K(A) \oplus \operatorname{Ann}_{A^{*}}\left(P_{n}(Z(A))\right) /\left[A, A^{*}\right]$.

Proof. Most assertions have been proved in [3] and the rest are easy to verify.

Corollary 8.2. Let $A$ and $B$ be two indecomposable finite dimensional algebras defined over an algebraically closed field of characteristic $p>0$. Suppose that $A$ and $B$ are stably equivalent of Morita type and that $A$ is symmetric. Then the condition that $\mathbb{T}(A)$ and $\mathbb{T}(B)$ are also stably equivalent of Morita type implies that the Auslander-Reiten conjecture holds for $A$ and $B$, that is, they have the same number of isoclasses of non-projective simple modules.

Proof. Since $A$ is symmetric, for any $n \geq 0$,

$$
\operatorname{dim} \operatorname{Ann}_{A^{*}}\left(P_{n}(A)\right)=\operatorname{dim} A / P_{n}(A)=\operatorname{dim} T_{n}(A) / K(A) .
$$

By Corollary 4.6(2), for any $n \geq 0, \operatorname{dim} T_{n}(A) / K(A)=\operatorname{dim} T_{n}(B) / K(B)$. On the other hand, Proposition [5.8(1) implies that for any $n \geq 0, \operatorname{dim} P_{n}^{\perp}(\mathbb{T}(A))=$ $\operatorname{dim} P_{n}^{\perp}(\mathbb{T}(B))$. By Proposition $8.1(9)$, we obtain that $\operatorname{dim} Z(A)=\operatorname{dim} Z(B)$ and this implies the Auslander-Reiten conjecture by Theorem 1.1 of [24].

This motivates the following

Question 8.3. Let $A$ and $B$ be two indecomposable, non-simple finite dimensional algebras which are stably equivalent of Morita type. Are their trivial extensions algebras $\mathbb{T}(A)$ and $\mathbb{T}(B)$ also stably equivalent of Morita type?

Although we feel that the answer should be negative, we do not know of a counterexample.

\section{Stable CyClic homology}

In this section we study the invariance of cyclic homology under stable equivalences of Morita type. The case of derived equivalences was considered by Keller in 16] using transfer maps. We shall adopt the same approach, but we work in the 
setup of ordinary algebras. For basic notions about cyclic homology, we refer the reader to [25] or, for a very readable brief introduction, [14.

Let $k$ be a commutative ring with unit. We shall write $\otimes$ instead of $\otimes_{k}$. In order to agree with the usual notation in cyclic homology, we shall modify our notation. We shall use $R, T, \ldots$ to denote $k$-algebras. Given a $k$-algebra $R$, the cyclic homology group $H C_{n}(R)$ is defined to be the homology of the total complex of the following double complex $C C_{\bullet \bullet}(R)$ :

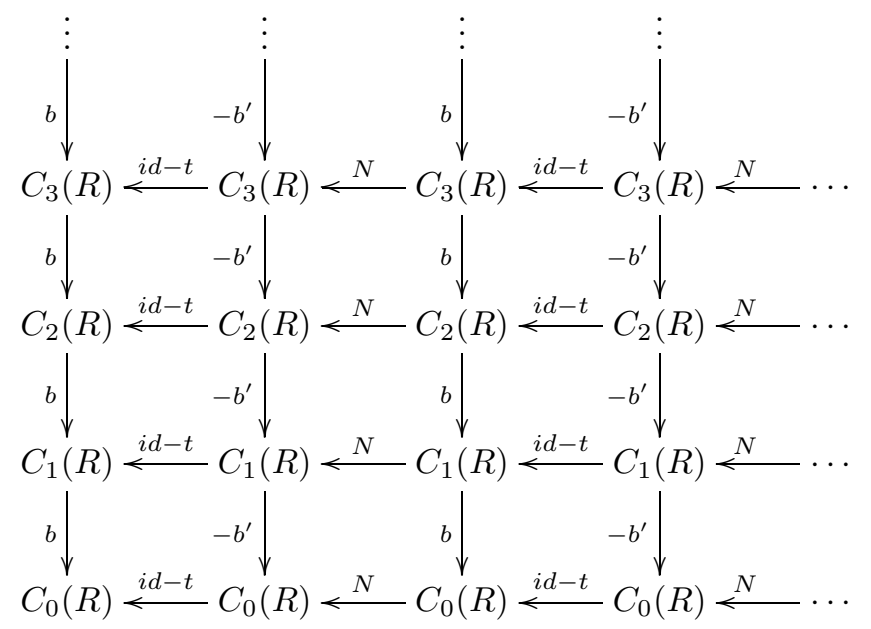

We recall the construction of the above double complex. Let $\left(C_{\bullet}^{\prime}(R), b^{\prime}\right)$ be the Bar complex. Namely, for $n \geq 0, C_{n}^{\prime}(R)=R^{\otimes(n+2)}$ and for $n \geq 0$ the differential $b^{\prime}: C_{n}^{\prime}(R) \rightarrow C_{n-1}^{\prime}(R)$ sends $x_{0} \otimes x_{1} \otimes \cdots \otimes x_{n+1}$ with $x_{0}, \ldots, x_{n+1} \in R$ to

$$
\sum_{i=0}^{n}(-1)^{i} x_{0} \otimes \cdots \otimes x_{i} x_{i+1} \otimes \cdots \otimes x_{n+1} .
$$

Note that here we use $b^{\prime}$ to denote the differential. Let $\left(C_{\bullet}(R), b\right)$ be the Hochschild complex. Namely, for $n \geq 0, C_{n}(R)=C_{n-1}^{\prime}(R)=R^{\otimes(n+1)}$ and for $n \geq 1$ the differential $b: C_{n}(R) \rightarrow C_{n-1}(R)$ sends $x_{0} \otimes x_{1} \otimes \cdots \otimes x_{n}$ with $x_{0}, \ldots, x_{n} \in R$ to

$$
\sum_{i=0}^{n-1}(-1)^{i} x_{0} \otimes \cdots \otimes x_{i} x_{i+1} \otimes \cdots \otimes x_{n}+(-1)^{n} x_{n} x_{0} \otimes x_{1} \otimes \cdots \otimes x_{n-1} .
$$

Note that here we use $b$ to denote the differential. For $n \geq 0$, consider the endomorphism $t$ of $C_{n}(R)=R^{\otimes(n+1)}$ defined for $x_{0}, \ldots, x_{n} \in R$ by

$$
t\left(x_{0} \otimes \cdots \otimes x_{n}\right)=(-1)^{n} x_{n} \otimes x_{0} \otimes \cdots \otimes x_{n-1} .
$$

In the diagram, we denote by $N$ the associated norm map

$$
N=i d+t+\cdots+t^{n}: C_{n}(R) \rightarrow C_{n}(R) .
$$

One can verify that $b(i d-t)=(i d-t) b^{\prime}$ and $N b=b^{\prime} N$. Thus $C C_{\bullet \bullet}(R)$ is a double complex. We define, for $n \geq 0$, another map $s: C_{n}(R) \rightarrow C_{n+1}(R)$ by

$$
s\left(x_{0} \otimes \cdots \otimes x_{n}\right)=1 \otimes x_{0} \otimes \cdots \otimes x_{n} .
$$

Now the Connes' operator $B: C_{n}(R) \rightarrow C_{n+1}(R)$ is defined to be

$$
B=(i d-t) s N: C_{n}(R) \rightarrow C_{n+1}(R) .
$$


Since it can be shown that $B^{2}=B b+b B=0, B$ induces a map $H H_{n}(R) \rightarrow$ $H H_{n+1}(R)$, still denoted by $B$.

The cyclic homology is defined to be $H C_{n}(R):=H_{n}\left(\operatorname{Tot}\left(C C_{\bullet \bullet}(R)\right)\right)$ for $n \geq 0$. It is easy to see that the bicomplex formed by the left-most two columns, denoted by $C C_{\bullet}^{\{2\}}$, has a total complex which is quasi-isomorphic to the Hochschild complex $(C \bullet(R), b)$. One has a short exact sequence of bicomplexes,

$$
0 \rightarrow C_{\bullet \bullet}\{2\}(R) \stackrel{I}{\rightarrow} C C_{\bullet \bullet}(R) \stackrel{S}{\rightarrow} C C_{\bullet \bullet}(R)[2,0] \rightarrow 0,
$$

where for the bicomplex $C C_{\bullet \bullet}(R),\left(C C_{\bullet \bullet}(R)[2,0]\right)_{p q}=C C_{p-2, q}(R)$. We thus have a long exact sequence, which is called Connes' long exact sequence,

$$
\cdots \stackrel{\partial}{\rightarrow} H H_{n}(R) \stackrel{I}{\rightarrow} H C_{n}(R) \stackrel{S}{\rightarrow} H C_{n-2}(R) \stackrel{\partial}{\rightarrow} H H_{n-1}(R) \stackrel{I}{\rightarrow} H C_{n-1}(R) \stackrel{S}{\rightarrow} \cdots
$$

The degree -2 map $S$ is called the periodicity map and we use $\partial$ to denote the connecting morphism instead of the usual $B$ to avoid possible confusion. As a consequence of this exact sequence, one has $H H_{0}(R) \cong H C_{0}(R)$ and

$$
H C_{n}(k)= \begin{cases}k & \text { if } n \text { is even } \\ 0 & \text { if } n \text { is odd }\end{cases}
$$

Now let $R$ and $T$ be two $k$-algebras. Let $M$ be an $R$-T-bimodule, which is finitely generated and projective as the right $T$-module. Then one can also define a transfer map $t_{M}: H C_{n}(R) \rightarrow H C_{n}(T)$ for $n \geq 0$ as in the case of Hochschild homology. The construction was given in [16, and also indicated in [4, Section 4.4]. The construction goes as follows. We begin with the transfer maps for the Hochschild complex $t_{M}: C_{\bullet}(R) \rightarrow C_{\bullet}(T)$ and prove that it commutes with the operators $b^{\prime}, b$ and $t$ (see Section 2). This provides a morphism from the bicomplex $C C_{\bullet \bullet}(R)$ to $C C_{\bullet . .}(T)$ which induces the desired map $t_{M}: H C_{n}(R) \rightarrow H C_{n}(T)$.

We include the proof of the following

Lemma 9.1. With the assumptions above, for $n \geq 0$, there are commutative diagrams,
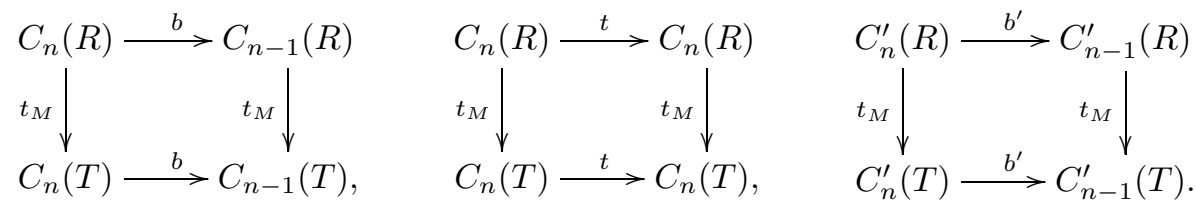

As a consequence, there is a morphism of bicomplexes $t_{M}: C_{\bullet \bullet}(R) \rightarrow C_{\bullet \bullet}(T)$ and thus $t_{M}: H C_{n}(R) \rightarrow H C_{n}(T)$.

Proof. We prove the commutativity of the first diagram, the proofs of the second and the third being similar. Since $M_{T}$ is finitely generated and projective, there are $x_{i} \in M$ and $\varphi_{i} \in \operatorname{Hom}_{T}(M, T)$ such that for each $x \in M$, we have $x=\sum_{i} x_{i} \varphi_{i}(x)$. 
For $a_{0}, \ldots, a_{n} \in R$, we have

$$
\begin{array}{rl}
t_{M} & b\left(a_{0} \otimes \cdots \otimes a_{n}\right) \\
= & \sum_{i=0}^{n-1}(-1)^{i} t_{M}\left(a_{0} \otimes \cdots \otimes a_{i} a_{i+1} \otimes \cdots \otimes a_{n}\right) \\
& +(-1)^{n} t_{M}\left(a_{n} a_{0} \otimes a_{1} \otimes \cdots \otimes a_{n-1}\right) \\
= & \sum_{i=0}^{n-1}(-1)^{i} \sum_{s_{0}, \ldots, s_{i}, s_{i+2}, \ldots, s_{n}} \varphi_{s_{0}}\left(a_{0} x_{s_{1}}\right) \otimes \cdots \otimes \varphi_{s_{i-1}}\left(a_{i-1} x_{s_{i}}\right) \\
& \otimes \varphi_{s_{i}}\left(a_{i} a_{i+1} x_{s_{i+2}}\right) \otimes \varphi_{s_{i+2}}\left(a_{i+2} x_{s_{i+3}}\right) \otimes \cdots \otimes \varphi_{s_{n}}\left(a_{n} x_{s_{0}}\right) \\
& +(-1)^{n} \sum_{s_{n}, s_{1}, \ldots, s_{n-1}} \varphi_{s_{n}}\left(a_{n} a_{0} x_{s_{1}}\right) \otimes \cdots \otimes \varphi_{s_{n-1}}\left(a_{n-1} x_{s_{n}}\right)
\end{array}
$$

and

$$
\begin{aligned}
& b t_{M}\left(a_{0} \otimes \cdots \otimes a_{n}\right) \\
& =\sum_{t_{0}, \ldots, t_{n}} b\left(\varphi_{t_{0}}\left(a_{0} x_{t_{1}}\right) \otimes \cdots \otimes \varphi_{t_{n}}\left(a_{n} x_{t_{0}}\right)\right) \\
& =\sum_{t_{0}, \ldots, t_{n}} \sum_{i=0}^{n-1}(-1)^{i} \varphi_{t_{0}}\left(a_{0} x_{t_{1}}\right) \otimes \cdots \otimes \varphi_{t_{i-1}}\left(a_{i-1} x_{t_{i}}\right) \\
& \otimes \varphi_{t_{i}}\left(a_{i} x_{t_{i+1}}\right) \varphi_{t_{i+1}}\left(a_{i+1} x_{t_{i+2}}\right) \otimes \varphi_{t_{i+2}}\left(a_{i+2} x_{t_{i+3}}\right) \otimes \cdots \otimes \varphi_{t_{n}}\left(a_{n} x_{t_{0}}\right) \\
& +\sum_{t_{0}, \ldots, t_{n}}(-1)^{n} \varphi_{t_{n}}\left(a_{n} x_{t_{0}}\right) \varphi_{t_{0}}\left(a_{0} x_{t_{1}}\right) \otimes \cdots \otimes \varphi_{t_{n-1}}\left(a_{n-1} x_{t_{n}}\right) \\
& =\sum_{i=0}^{n-1}(-1)^{i} \sum_{t_{0}, \ldots, t_{i}, t_{i+2}, \ldots, t_{n}} \varphi_{t_{0}}\left(a_{0} x_{t_{1}}\right) \otimes \cdots \otimes \varphi_{t_{i-1}}\left(a_{i-1} x_{t_{i}}\right) \\
& \otimes\left(\sum_{t_{i+1}} \varphi_{t_{i}}\left(a_{i} x_{t_{i+1}}\right) \varphi_{t_{i+1}}\left(a_{i+1} x_{t_{i+2}}\right)\right) \otimes \cdots \otimes \varphi_{t_{n}}\left(a_{n} x_{t_{0}}\right) \\
& +(-1)^{n} \sum_{t_{1}, \ldots, t_{n}}\left(\sum_{t_{0}} \varphi_{t_{n}}\left(a_{n} x_{t_{0}}\right) \varphi_{t_{0}}\left(a_{0} x_{t_{1}}\right)\right) \otimes \cdots \otimes \varphi_{t_{n-1}}\left(a_{n-1} x_{t_{n}}\right) \\
& =\sum_{i=0}^{n-1}(-1)^{i} \sum_{t_{0}, \ldots, t_{i}, t_{i+2}, \ldots, t_{n}} \varphi_{t_{0}}\left(a_{0} x_{t_{1}}\right) \otimes \cdots \otimes \varphi_{t_{i-1}}\left(a_{i-1} x_{t_{i}}\right) \\
& \otimes \varphi_{t_{i}}\left(a_{i} a_{i+1} x_{t_{i+2}}\right) \otimes \varphi_{t_{i+2}}\left(a_{i+2} x_{t_{i+3}}\right) \otimes \cdots \otimes \varphi_{t_{n}}\left(a_{n} x_{t_{0}}\right) \\
& +(-1)^{n} \sum_{t_{1}, \ldots, t_{n}} \varphi_{t_{n}}\left(a_{n} a_{0} x_{t_{1}}\right) \otimes \varphi_{t_{1}}\left(a_{1} x_{t_{2}}\right) \otimes \cdots \otimes \varphi_{t_{n-1}}\left(a_{n-1} x_{t_{n}}\right),
\end{aligned}
$$

where the last equality uses two other equalities, which are true by definition,

$$
\begin{aligned}
& \sum_{t_{i+1}} \varphi_{t_{i}}\left(a_{i} x_{t_{i+1}}\right) \varphi_{t_{i+1}}\left(a_{i+1} x_{t_{i+2}}\right) \\
& =\varphi_{t_{i}}\left(\sum_{t_{i+1}} a_{i} x_{t_{i+1}} \varphi_{t_{i+1}}\left(a_{i+1} x_{t_{i+2}}\right)\right)=\varphi_{t_{i}}\left(a_{i} a_{i+1} x_{t_{i+2}}\right)
\end{aligned}
$$


and

$$
\begin{aligned}
& \sum_{t_{0}} \varphi_{t_{n}}\left(a_{n} x_{t_{0}}\right) \varphi_{t_{0}}\left(a_{0} x_{t_{1}}\right) \\
& \quad=\varphi_{t_{n}}\left(\sum_{t_{0}} a_{n} x_{t_{0}} \varphi_{t_{0}}\left(a_{0} x_{t_{1}}\right)\right)=\varphi_{t_{n}}\left(a_{n} a_{0} x_{t_{1}}\right) .
\end{aligned}
$$

We thus have

$$
t_{M} b\left(a_{0} \otimes \cdots \otimes a_{n}\right)=b t_{M}\left(a_{0} \otimes \cdots \otimes a_{n}\right) .
$$

Corollary 9.2. With the assumptions above, there is a commutative diagram of exact sequences of bicomplexes

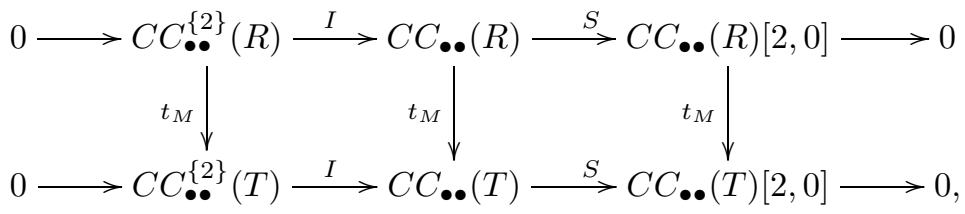

and hence there is the following exact commutative diagram:

$$
\begin{aligned}
& \ldots \stackrel{S}{\rightarrow} H C_{n-1}(R) \stackrel{\partial}{\rightarrow} H H_{n}(R) \stackrel{I}{\rightarrow} H C_{n}(R) \stackrel{S}{\rightarrow} H C_{n-2}(R) \stackrel{\partial}{\rightarrow} H H_{n-1}(R) \stackrel{I}{\rightarrow} H C_{n-1}(R) \stackrel{S}{\longrightarrow} \ldots
\end{aligned}
$$

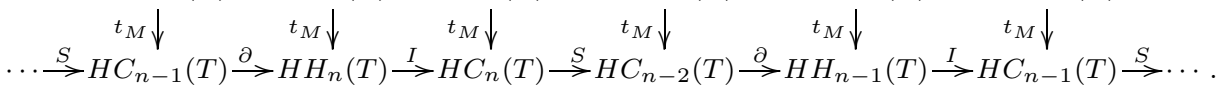

Corollary 9.3. With the assumptions above, there is a commutative diagram for any $n \geq 0$,

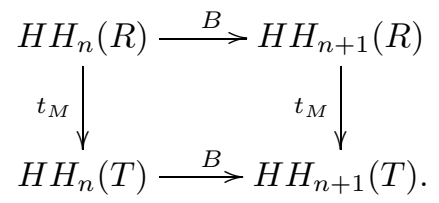

Now we list some properties of the new transfer maps on cyclic homology.

Proposition 9.4. Let $R, T$ and $U$ be $k$-algebras.

(1) If $M$ is an $R$-T-bimodule and $N$ is a $T$-U-bimodule such that $M_{T}$ and $N_{U}$ are finitely generated and projective, then there is an equality $t_{N} \circ t_{M}=t_{M \otimes_{T} N}$ : $H C_{n}(R) \rightarrow H C_{n}(U)$, for each $n \geq 0$.

(2) Let

$$
0 \rightarrow L \rightarrow M \rightarrow N \rightarrow 0
$$

be a short exact sequence of $R$-T-bimodules which are finitely generated and projective as right $T$-modules. Then $t_{M}=t_{L}+t_{N}: H C_{n}(R) \rightarrow H C_{n}(T)$, for each $n \geq 0$.

(3) Let $k$ be an algebraically closed field, and let $R$ and $T$ be finite dimensional $k$-algebras. For a finitely generated projective $R$-T-bimodule $P$, the transfer map $t_{P}: H C_{n}(R) \rightarrow H C_{n}(T)$ is zero for $n$ odd.

(4) Consider $R$ as an $R$-R-bimodule by left and right multiplication, then $t_{R}$ : $H C_{n}(R) \rightarrow H C_{n}(R)$ is the identity map for any $n \geq 0$. 
Proof. The assertions (1), (2) and (4) follow from the corresponding statements for transfer maps between Hochschild homology groups. Let us prove the assertion (3). Recall that $H C_{n}(k)=k$ if $n$ is even and $H C_{n}(k)=0$ if $n$ is odd. Since $k$ is an algebraically closed field, one can assume (without loss of generality) that $P=R e \otimes f T$ for certain idempotents $e \in R$ and $f \in T$. By (1), $t_{P}=t_{R e} \circ t_{f T}$ : $H C_{n}(R) \rightarrow H C_{n}(k) \rightarrow H C_{n}(T)$. The assertion (3) thus follows.

Remark 9.5. As in the case of Hochschild homology, for a bounded (cochain) complex $X^{\bullet}$ of $R$-T-bimodules whose terms are finitely generated and projective as right $T$-modules, one can define a transfer map $t_{X} \bullet: H C_{n}(R) \rightarrow H C_{n}(T)$ by $t_{X} \bullet:=\sum_{i}(-1)^{i} t_{X^{i}}$. Note that if $Y^{\bullet}$ is another bounded complex of $R$ - $T$-bimodules whose terms are finitely generated and projective as right $T$-modules such that $X^{\bullet}$ and $Y^{\bullet}$ are quasi-isomorphic, then $t_{X} \bullet=t_{Y} \bullet$

Theorem 9.6. Let $k$ be an algebraically closed field of arbitrary characteristic and let $R$ and $T$ be two finite dimensional $k$-algebras which are stably equivalent of Morita type.

(1) For $n>0$ odd, $\operatorname{dim} H C_{n}(R)=\operatorname{dim} H C_{n}(T)$.

(2) Suppose that $R$ and $T$ have no semisimple direct summands. Then for any $n \geq 0$ even, the following statements are equivalent:

(i) the Auslander-Reiten conjecture holds for this stable equivalence of Morita type;

(ii) $\operatorname{dim} H C_{n}(R)=\operatorname{dim} H C_{n}(T)$.

Proof. Suppose that two bimodules $M$ and $N$ define a stable equivalence of Morita type between $R$ and $T$ by $M \otimes_{T} N \simeq R \oplus P, N \otimes_{R} M \simeq T \oplus Q$. Since $t_{R}=1_{H C_{n}(R)}$ and $t_{T}=1_{H C_{n}(T)}$, the transfer maps $t_{M}: H C_{n}(R) \rightarrow H C_{n}(T)$ and $t_{N}: H C_{n}(T) \rightarrow$ $H C_{n}(R)$ are mutually inverse group isomorphisms for all $n>0$ by Proposition 9.4 . This proves the first statement.

For the second statement, one uses the long exact sequence connecting Hochschild homology and cyclic homology. By Corollary 9.2 , for $n \geq 2$ even, we have a commutative diagram,

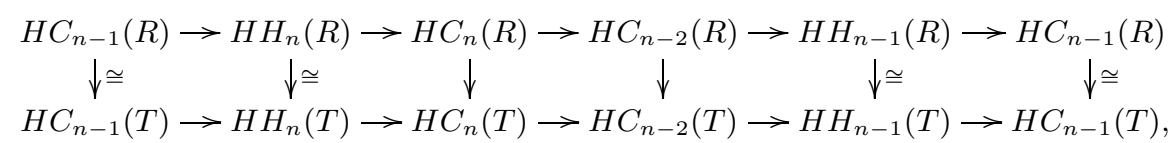

where the second and the third isomorphisms are deduced from [24, Remark 3.3] and where the second (and hence the fourth) follows from (1). We thus have by 5-lemma that $H C_{n}(R) \cong H C_{n}(T)$ if and only if $H C_{n-2}(R) \cong H C_{n-2}(T)$, but $H C_{0}(R) \cong H H_{0}(R)$ and by Theorem 1.1 of [24], $H H_{0}(R) \cong H H_{0}(T)$ is equivalent to the Auslander-Reiten conjecture. We are done.

By the previous theorem, one can define a stable version of cyclic homology for a finite dimensional algebra over a field.

Definition 9.7. Let $R$ be a finite dimensional algebra over a field $k$. The stable cyclic homology of $R$, in degree $n$, is defined to be

$$
H C_{n}^{s t}(R)=\bigcap_{P} \operatorname{Ker}\left(t_{P}: H C_{n}(R) \rightarrow H C_{n}(k)\right),
$$

where $P$ runs through the set of isomorphism classes of finite dimensional left projective $R$-modules (which here are considered as $R$ - $k$-bimodules). 
Obviously $H C_{n}^{s t}(R)$ differs from $H C_{n}(R)$ only when $n$ is even. In Connes' long exact sequence one can replace $H C_{n}(R)$ by $H C_{n}^{s t}(R)$ and $H H_{0}(R)$ by $H H_{0}^{s t}(R)$. This is the content of the following

Proposition 9.8. There is a long exact sequence

$$
\cdots \stackrel{\partial}{\rightarrow} H H_{n}^{s t}(R) \stackrel{I}{\rightarrow} H C_{n}^{s t}(R) \stackrel{S}{\rightarrow} H C_{n-2}^{s t}(R) \stackrel{\partial}{\rightarrow} H H_{n-1}^{s t}(R) \stackrel{I}{\rightarrow} H C_{n-1}^{s t}(R) \stackrel{S}{\rightarrow} \cdots .
$$

If $M$ is an $R$-T-bimodule such that $M_{T}$ is finitely generated and projective, then the following diagram commutes.

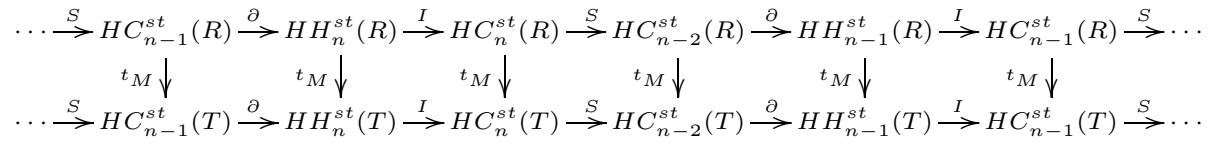

Proof. We have the following commutative diagram with exact columns and whose lower two rows are also exact,

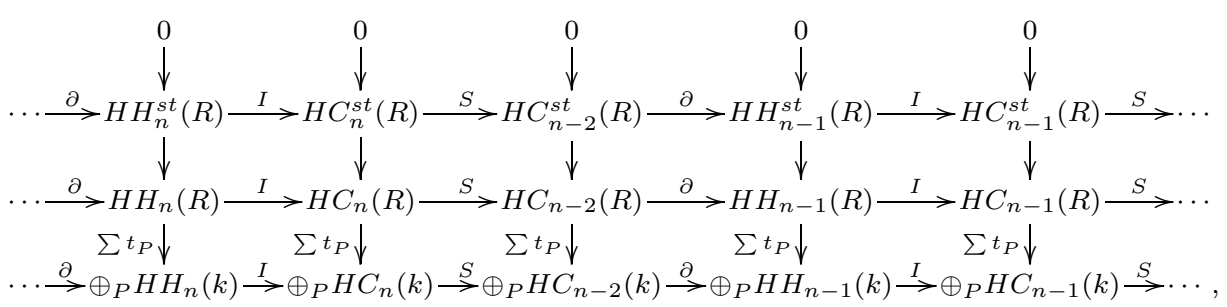

where $P$ runs through the set of isoclasses of finite dimensional left projective $R$ modules. We need to show that the first row is exact as well. This can be done by diagram chasing, using the facts that $H C_{n}(k)=\left\{\begin{array}{ll}k & \text { if } n \text { is even, } \\ 0 & \text { if } n \text { is odd. }\end{array}\right.$ and that $H H_{n}(k)=\left\{\begin{array}{cc}k & \text { if } n=0 \\ 0 & \text { otherwise. }\end{array}\right.$

Theorem 9.9. The stable cyclic homology is invariant under derived equivalences and stable equivalences of Morita type.

Proof. The invariance of the stable cyclic homology under stable equivalences of Morita type is easy by Definition 9.7 and Proposition 9.4 (refer to 24, Theorem 4.7]). The case of derived equivalence is a consequence of the commutative diagram in Proposition 9.8 (we only need to replace $t_{M}$ by $t_{X} \bullet$, where $X^{\bullet}$ is a two-sided tilting complex; note that $t_{X} \cdot$ gives isomorphisms between stable Hochschild homology groups) and the fact that $H C_{0}^{s t} \cong H H_{0}^{s t}$.

Remark 9.10. As in the case of ordinary cyclic homology, transfer maps can also be defined over negative cyclic homology and periodic cyclic homology. We leave the details to the reader.

To conclude this section, we add some comments on transfer maps in cyclic cohomology and its variants. 
Let $k$ be a commutative ring with unit and let $R$ be a $k$-algebra. The cyclic cohomology is defined to be the cohomology of the total complex of $C C^{\bullet \bullet}(R):=$ $\operatorname{Hom}_{k}\left(C C_{\bullet \bullet}(R), k\right)$. If we have another $k$-algebra $T$ and an $R$-T-bimodule $M$ such that $M_{T}$ is finitely generated and projective, then we have the transfer map defined as above $t_{M}: C C_{\bullet \bullet}(R) \rightarrow C C_{\bullet \bullet}(T)$ which in turn induces a map $t^{M}: C C^{\bullet \bullet}(T) \rightarrow$ $C C^{\bullet \bullet}(R)$. This new chain map induces a map in cyclic cohomology, denoted also by $t^{M}: H C^{n}(T) \rightarrow H H^{n}(R)$ and called a transfer map in cyclic cohomology. Obviously, these new maps have some properties like those of Proposition 9.4. Evidently, we can also define transfer maps over negative cyclic cohomology and periodic cyclic cohomology. Now let $R$ be a finite dimensional algebra over a field. For $n \geq 0$, the stable cyclic cohomology $H C_{s t}^{n}(R)$ is defined to be the quotient of $H C^{n}(R)$ by the sum of the images of $t^{P}: H C^{n}(k) \rightarrow H C^{n}(R)$, where $P$ runs through the set of isoclasses of (finitely generated) projective $R$-modules. We can also define stable versions of negative cyclic cohomology and periodic cyclic cohomology. We also have similar results as Proposition 9.8 and Theorem 9.9.

\section{Batalin-Vilkovisky vs Gerstenhaber}

Recall the definition of Gerstenhaber algebras and Batalin-Vilkovisky algebras. Let $k$ be a commutative ring with unit.

Definition 10.1. A Gerstenhaber algebra is a graded $k$-module $A=\bigoplus_{i \in \mathbb{Z}} A^{i}$ equipped with two linear maps: a cup product

$$
\bigcup: A^{i} \otimes A^{j} \rightarrow A^{i+j}, x \otimes y \mapsto x \cup y
$$

and a Lie bracket of degree -1

$$
[,]: A^{i} \otimes A^{j} \rightarrow A^{i+j-1}, x \otimes y \mapsto[x, y]
$$

such that

(a) the cup product $\bigcup$ makes $A$ into a graded commutative algebra;

(b) the Lie bracket $[-,-]$ gives $A$ a structure of graded Lie algebra of degree -1 . This means that for homogeneous elements $a, b, c \in A$,

$$
[a, b]=-(-1)^{(|a|-1)(|b|-1)}[b, a]
$$

and

$$
(-1)^{(|a|-1)(|c|-1)}[[a, b], c]+(-1)^{(|b|-1)(|a|-1)}[[b, c], a]+(-1)^{(|c|-1)(|b|-1)}[[c, a], b]=0,
$$

where $|a|$ denotes the degree of $a$;

(c) the cup product and the Lie bracket satisfy the Poisson rule, which means that for any $c \in A^{|c|}$ the adjunction map $[-, c]: A^{i} \rightarrow A^{i+|c|-1}, a \mapsto[a, c]$ is a $(|c|-1)$-derivation, i.e., for homogeneous $a, b \in A$,

$$
[a \cup b, c]=[a, c] \cup b+(-1)^{|a|(|c|-1)} a \cup[b, c] .
$$

Definition 10.2. A Batalin-Vilkovisky (BV) algebra is a Gerstenhaber algebra $A$ together with a degree -1 operator $\Delta: A^{\bullet} \rightarrow A^{\bullet-1}$ satisfying $\Delta \circ \Delta=0$ and

$$
[a, b]=-(-1)^{(|a|-1)|b|}\left(\Delta(a \cup b)-(\Delta a) \cup b-(-1)^{|a|} a \cup(\Delta b)\right)
$$

for $a, b \in A$. 
The first examples of Gerstenhaber algebras are Hochschild cohomology algebras of rings first discovered by Gerstenhaber in [11. We recall his construction. Let $R$ be a $k$-algebra. Let $f \in C^{n}(R)=\operatorname{Hom}_{k}\left(R^{\otimes n}, R\right)$ and $g \in C^{m}(R)$ with $n, m \geq 0$. If $n, m \geq 1$, then for $1 \leq i \leq n$, define

$$
\begin{aligned}
& \left(f \circ_{i} g\right)\left(a_{1} \otimes \cdots \otimes a_{n+m-1}\right) \\
& \quad=f\left(a_{1} \otimes \cdots \otimes a_{i-1} \otimes g\left(a_{i} \otimes \cdots \otimes a_{i+m-1}\right) \otimes a_{i+m} \otimes \cdots \otimes a_{n+m-1}\right) ;
\end{aligned}
$$

if $n \geq 1$ and $m=0$, then $g \in A$ and for $1 \leq i \leq n$, define

$$
\left(f \circ_{i} g\right)\left(a_{1} \otimes \cdots \otimes a_{n-1}\right)=f\left(a_{1} \otimes \cdots \otimes a_{i-1} \otimes g \otimes a_{i} \otimes \cdots \otimes a_{n-1}\right) ;
$$

for any other case, define $f \circ_{i} g$ to be zero. Now denote

$$
f \circ g=\sum_{i=1}^{n}(-1)^{(n-1)(i-1)} f \circ_{i} g
$$

and

$$
[f, g]=f \circ g-(-1)^{(n-1)(m-1)} g \circ f .
$$

The above [, ] is just the Gerstenhaber Lie bracket over the Hochschild cohomology algebra. This Lie bracket with the usual cup product makes the Hochschild cohomology algebra into a Gerstenhaber algebra.

Tradler noticed that the Hochschild cohomology algebra of a symmetric algebra is a BV algebra in [34. This fact has been reproved by many authors ([26, 10] etc.). For a symmetric algebra $R$, Tradler showed that the operator $\Delta$ over Hochschild cohomology corresponds to the Connes' $B$ operator on Hochschild homology via the duality between Hochschild cohomology and homology. Our main result in this section is that a derived equivalence between symmetric algebras preserves the structure of BV algebras of the Hochschild cohomology algebras.

Theorem 10.3. Let $k$ be a field. Let $R$ and $T$ be two symmetric algebras which are derived equivalent, by an equivalence given by a two-sided tilting complex ${ }_{R} X_{T}^{\bullet}$ whose terms are bimodules that are projective as left $R$-modules and right $T$-modules. Then the transfer map $t^{X^{\bullet}}: H H^{*}(T) \rightarrow H H^{*}(R)$ is an isomorphism of $B V$-algebras.

Proof. We need to show that transfer maps in Hochschild cohomology commute with the operator $\Delta$. By the compatibility theorem, Theorem 2.10, we only need to show that transfer maps in Hochschild homology commute with Connes' $B$ operator, but this is Corollary 9.3 .

As a consequence, we obtain a special case of a theorem of Keller (16]) which says that a derived equivalence preserves the structure of Gerstenhaber algebra structure over the Hochschild cohomology algebras.

Corollary 10.4. With the above assumptions, the transfer map $t^{X^{\bullet}}: H H^{*}(T) \rightarrow$ $H H^{*}(R)$ is an isomorphism of Gerstenhaber algebras.

Menichi ([26, Corollary 1.7]) also proved that the negative cyclic cohomology of a symmetric algebra is a graded Lie algebra of degree -2 . His construction is as follows. Let $R$ be a $k$-algebra. Then we have the long exact sequence

$$
\cdots \stackrel{S}{\rightarrow} H C_{-}^{n+1}(R) \stackrel{\partial}{\rightarrow} H H^{n}(R) \stackrel{I}{\rightarrow} H C_{-}^{n}(R) \stackrel{S}{\rightarrow} H C_{-}^{n+2}(R) \stackrel{\partial}{\rightarrow} \cdots .
$$

Let $a \in H C_{-}^{n}(R)$ and $b \in H C_{-}^{m}(R)$. Then the Lie bracket on $H C_{-}^{*}(R)$ is defined as

$$
[a, b]=(-1)^{|a|} I(\partial a \cup \partial b) .
$$


Theorem 10.5. Let $R$ and $T$ be two symmetric algebras which are related by a derived equivalence that is given by ${ }_{R} X_{T}^{\bullet}$, a two-sided tilting complex whose terms are bimodules that are projective as left $R$-modules and right $T$-modules. Then the transfer map $t^{X^{\bullet}}: H C_{-}^{*}(T) \rightarrow H C_{-}^{*}(R)$ is an isomorphism of graded Lie algebras.

Proof. This follows from Proposition 3.6 and the analogue of Corollary 9.2 for negative cyclic cohomology.

Now we consider the invariance of the above structures under a stable equivalence of Morita type. Let $k$ be a field and let $R$ be a symmetric $k$-algebra. Then by Corollary 9.3 , transfer maps commute with the operator $\Delta: H H^{n+1}(R) \rightarrow H H^{n}(R)$. Hence there is an induced map $\Delta_{s t}: H H_{s t}^{n+1}(R) \rightarrow H H_{s t}^{n}(R)$ for $n \geq 0$. This means that the stable Hochschild cohomology algebra of a symmetric algebra is still a BV algebra. This also proves that the projective centre of a symmetric algebra is a Lie ideal for the Gerstenhaber Lie bracket. We have thus proved the following

Lemma 10.6. Let $k$ be a field and let $R$ be a symmetric $k$-algebra. Then $H H_{s t}^{*}$ is a $B V$ algebra with the $\Delta$-operator induced from that of $H H^{*}$ and as a consequence, the projective centre is a Lie ideal for the Gerstenhaber Lie algebra structure over the Hochschild cohomology algebra.

Now for stable negative cyclic cohomology, we have a long exact sequence

$$
\cdots \stackrel{S}{\rightarrow} H C_{-, s t}^{n+1}(R) \stackrel{\partial}{\rightarrow} H H_{s t}^{n}(R) \stackrel{I}{\rightarrow} H C_{-, s t}^{n}(R) \stackrel{S}{\rightarrow} H C_{-, s t}^{n+2}(R) \stackrel{\partial}{\rightarrow} \cdots .
$$

Let $a \in H C_{-, s t}^{n}(R)$ and $b \in H C_{-, s t}^{m}(R)$. Then by [26, Lemma 7.2], we define a Lie bracket on $H C_{-, s t}^{*}(R)$ as

$$
[a, b]=(-1)^{|a|} I(\partial a \cup \partial b) .
$$

Combining Theorem 10.3, Theorem 10.5 and Lemma 10.6, we easily obtain the following

Theorem 10.7. Let $k$ be an algebraically closed field. Let $R$ and $T$ be two symmetric algebras which are related by a stable equivalence of Morita type that is given by ${ }_{R} M_{T}$ and ${ }_{T} N_{R}$, which are projective as $R$-modules and as T-modules. Suppose that the bilinear form on $R$ is induced from that of $T$. Then the transfer map $t^{M}: H H_{s t}^{*}(T) \rightarrow H H_{s t}^{*}(R)$ is an isomorphism of $B V$ algebras and $t^{M}$ : $H C_{-, s t}^{*}(T) \rightarrow H C_{-, s t}^{*}(R)$ is an isomorphism of graded Lie algebras.

Since stable Hochschild cohomology is invariant under a derived equivalence, similarly as in the preceding result, we have the following

Theorem 10.8. Let $k$ be an algebraically closed field. Let $R$ and $T$ be two symmetric algebras which are derived equivalent, where the equivalence is given by ${ }_{R} X_{T}^{\bullet}$ a tilting complex whose terms are bimodules that are projective as left $R$-modules and right $T$-modules. Then the transfer $\operatorname{map} t^{X^{\bullet}}: H H_{s t}^{*}(T) \rightarrow H H_{s t}^{*}(R)$ is an isomorphism of $B V$ algebras and $t^{X^{\bullet}}: H C_{-, s t}^{*}(T) \rightarrow H C_{-, s t}^{*}(R)$ is an isomorphism of graded Lie algebras.

\section{REFERENCES}

[1] F. W. Anderson and K. R. Fuller, Rings and categories of modules. Graduate Texts in Mathematics, Vol. 13. Springer-Verlag, New York-Heidelberg, 1974. MR0417223 (54:5281)

[2] M. Auslander, I. Reiten and S. O. Smalø, Representation theory of Artin algebras. Cambridge University Press, 1995. MR.1314422 (96c:16015) 
[3] C. Bessenrodt, T. Holm and A. Zimmermann, Generalized Reynolds ideals for non-symmetric algebras. J. Algebra 312 (2007), 985-994. MR2333196 (2008e:16001)

[4] S. Bouc, Bimodules, trace généralisée, et transfers en homologie de Hochschild. Preprint, 1997. Available on http://people.math.jussieu.fr/ bouc/

[5] T. Breuer, L. Héthelyi, E. Horváth. B. Külshammer and J. Murray, Cartan invariants and central ideals of group algebras, J. Algebra (3) 296 (2006), 177-195. MR2192603 (2006m:20016)

[6] M. Broué, Isométries parfaites, types de blocs, catégories dérivées. Astérisque 181-182 (1990), 61-92. MR 1051243 (91i:20006)

[7] M. Broué, Equivalences of blocks of group algebras. In: Finite dimensional algebras and related topics. V. Dlab and L. L. Scott (eds.), Kluwer, 1994, 1-26. MR.1308978 (97c:20004)

[8] D. Dugger and B. Shipley, K-theory and derived equivalences. Duke Math. J. 124 (2004), no. 3, 587-617. MR2085176 (2005e:19005)

[9] A. S. Dugas and R. Martinez-Villa, A note on stable equivalences of Morita type. J. Pure Appl. Algebra 208 (2007), no. 2, 421-433. MR2277684 (2007k:16012)

[10] C. H. Eu and T. Schedler, Calabi-Yau Frobenius algebras. J. Algebra 321 (2009), no. 3, 774-815. MR2488552 (2009m:16019)

[11] M. Gerstenhaber, The cohomology structure of an associative ring. Ann. of Math. (2) 78 (1963) 267-288. MR0161898 (28:5102)

[12] D. Happel, On the derived category of a finite-dimensional algebra, Comment. Math. Helv. 62 (1987), 339-389. MR910167 (89c:16029)

[13] D. Happel, Triangulated categories in the representation theory of finite dimensional algebras, LMS Lecture Note Series 119, Cambridge University Press, 1988. MR935124 (89e:16035)

[14] C. Kassel, Homology and cohomology of associative algebras - A concise introduction to cyclic homology, Notes of a course given in the Advanced School on Non-commutative Geometry at ICTP, Trieste in August 2004. Available at http://www-irma.u-strasbg.fr/ kassel/pubICTP04.html

[15] B. Keller, Deriving DG categories. Ann. Sci. École Norm. Sup. (4) 27 (1994), no. 1, 63-102. MR.1258406 (95e:18010)

[16] B. Keller, Invariance and localization for cyclic homology of DG algebras. J. Pure Appl. Algebra 123 (1998), 223-273. MR 1492902 (99c:16009)

[17] S. König and A. Zimmermann, Derived equivalences for group rings. Lecture Notes in Mathematics 1685, Springer Verlag, Berlin-Heidelberg, 1998. MR1649837 (2000g:16018)

[18] B. Külshammer, Bemerkungen über die Gruppenalgebra als symmetrische Algebra I, II, III, $I V$, J. Algebra 72 (1981), 1-7; J. Algebra 75 (1982), 59-69; J. Algebra 88 (1984), 279-291; J. Algebra 93 (1985), 310-323.

[19] B. Külshammer, Group-theoretical descriptions of ring theoretical invariants of group algebras. Progress in Math. 95 (1991), 425-441. MR1112173 (92d:16037)

[20] M. Linckelmann, Transfer in Hochschild cohomology of blocks of finite groups. Algebras and Representation Theory 2 (1999), 107-135. MR 1702272 (2000h:20024)

[21] Y. M. Liu, On stable equivalences induced by exact functors. Proc. Amer. Math. Soc. 134 (2006), 1605-1613. MR2204270 (2006k:16027)

[22] Y. M. Liu, Summands of stable equivalences of Morita type. Comm. in Algebra 36(10) (2008), 3778-3782. MR 2458406 (2009m:16028)

[23] Y. M. Liu and C. C. Xi, Constructions of stable equivalences of Morita type for finite dimensional algebras II. Math. Z. 251 (2005), 21-39. MR2176462 (2007f:16029)

[24] Y. M. Liu, G. Zhou and A. Zimmermann Higman ideal, stable Hochschild homology and Auslander-Reiten conjecture, to appear in Math. Zeit., available at http://www .mathinfo.u-picardie.fr/alex/alexpapers.html.

[25] Jean-Louis Loday, Cyclic homology. Appendix E by Mara O. Ronco. Second edition. Chapter 13 by the author in collaboration with Teimuraz Pirashvili. Grundlehren der Mathematischen Wissenschaften [Fundamental Principles of Mathematical Sciences], (1998) 301. MR,1600246 (98h:16014)

[26] L. Menichi, Batalin-Vilkovisky algebras and cyclic cohomology of Hopf algebras. K-Theory 32 (2004), no. 3, 231-251. MR.2114167 (2006c:16018)

[27] Z. Pogorzaly, Invariance of Hochschild cohomology algebras under stable equivalences of Morita type. J. Math. Soc. Japan 53 (2001), no. 4, 913-918. MR1852888 (2002m:16007) 
[28] J. Rickard, Morita theory for derived categories, J. London Math. Soc. 39 (1989), 436-456. MR1002456 (91b:18012)

[29] J. Rickard, Derived categories and stable equivalence. J. Pure Appl. Algebra 61(3) (1989), 303-317. MR.1027750 (91a:16004)

[30] J. Rickard, Derived equivalences as derived functors. J. London Math. Soc. 43 (1991), 37-48. MR1099084 (92b:16043)

[31] J. Rickard, Some recent advances in modular representation theory. Algebras and modules, I (Trondheim, 1996), 157-178, CMS Conf. Proc., 23, Amer. Math. Soc., Providence, RI, 1998. MR $1648606(99 \mathrm{~h}: 20011)$

[32] J. J. Rotman, An introduction to homological algebra. Pure and Applied Mathematics, 85. Academic Press, Inc. Harcourt Brace Jovanovich, Publishers, New York-London, 1979. MR538169 (80k:18001)

[33] R. W. Thomason and T. F. Trobaugh, Higher algebraic $K$-theory of schemes and of derived categories. In:The Grothendieck Festschrift (a collection of papers to honor Grothendieck's 60th birthday) vol. 3, Birkhauser, 1990, 247-435. MR1106918 (92f:19001)

[34] T. Tradler, The Batalin-Vilkovisky algebra on Hochschild cohomology induced by infinity inner products. Ann. Inst. Fourier (Grenoble) 58 (2008), no. 7, 2351-2379. MR2498354 (2010a:16020)

[35] A. Zimmermann, Invariance of generalized Reynolds ideals under derived equivalences. Mathematical Proceedings of the Royal Irish Academy 107A(1) (2007), 1-9. MR2289795 (2008a:16010)

[36] A. Zimmermann, Fine Hochschild invariants of derived categories for symmetric algebras, J. Algebra 308 (2007) 350-367. MR2290926 (2007k:16018)

[37] A. Zimmermann, Hochschild homology invariants of Külshammer type of derived categories, preprint (2007) available at http://www.mathinfo.u-picardie.fr/alex/alexpapers.html. MR2290926 (2007k:16018)

Mathematisches Institut, Universität zU Köln, Weyertal 86-90, D-50931 Köln, GerMANY

E-mail address: skoenig@mi.uni-koeln.de

Current address: Institut für Algebra und Zahlentheorie, Universtät Stuttgart, Pfaffenwaldring 57, D-70569, Stuttgart, Germany

E-mail address: skoenig@mathmatik.uni-stuttgart.de

School of Mathematical Sciences, Beijing Normal University, Beijing 100875, PeoPLE's Republic of China

E-mail address: ymliu@bnu.edu.cn

Institut für Mathematik, Universität Paderborn, 33098 Paderborn, Germany

E-mail address: gzhou@math.uni-paderborn.de

Current address: Ecole Polytechnique Fédérale de Lausanne, SB-MATHGEOM-CTG, Lausanne 1015, Switzerland

E-mail address: guodong.zhou@epfl.ch 